CONFORMAL GEOMETRY AND DYNAMICS

An Electronic Journal of the American Mathematical Society

Volume 10, Pages 288-325 (October 10, 2006)

S $1088-4173(06) 00134-2$

\title{
THE CORE CHAIN OF CIRCLES OF MASKIT'S EMBEDDING FOR ONCE-PUNCTURED TORUS GROUPS
}

\author{
IRENE SCORZA
}

\begin{abstract}
In this paper, we describe the limit set $\Lambda_{n}$ of a sequence of manifolds $N_{n}$ in the boundary of Maskit's embedding of the once-punctured torus. We prove that $\Lambda_{n}$ contains a chain of tangent circles $\left\{C_{n, j}\right\}$ that are described from the end invariants of the manifold. In particular, we give estimates in terms of $n$ of the radii $r_{n, j}$ of the circles and prove that $r_{n, j}$ decrease when $n$ tends to infinity. We then apply these results to McShane's identity, to obtain an estimate of the width of the limit set in terms of $n$.
\end{abstract}

\section{INTRODUCTION}

Let $G$ be a once-punctured torus group, i.e. a free, discrete two-generator group of Möbius transformations such that the commutator of the two generators $a, b$ is parabolic. Let $N$ be the hyperbolic 3-manifold obtained as the quotient of hyperbolic space by $G$. Then $G$ can be seen as $\rho\left(\pi_{1}(S)\right)$ where $S$ is a hyperbolic once-punctured torus and

$$
\rho: \pi_{1}(S) \longrightarrow P S L_{2}(\mathbb{C})
$$

is a representation. For a generic $N$, the limit set $\Lambda$ is a very complicated subset of the complex plane: For Fuchsian groups, $\Lambda$ is a circle; for quasi-Fuchsian groups, $\Lambda$ is topologically a circle, but on the boundary of a quasi-Fuchsian space, $\Lambda$ can be a complicated non-embedded Peano curve and it can also degenerate to the full complex plane. Under some assumptions, Bowditch [6], Minsky [21] and McMullen 18 proved that the limit set $\Lambda$ is the image of the limit set $\Lambda_{0}$ of a Fuchsian group by a continuous $\rho$-equivariant map called the Cannon-Thurston map for $G$.

In this paper, we describe and study the limit set $\Lambda_{n}$ of a sequence of manifolds $N_{n}$ in the boundary of the quasi-Fuchsian space. They are geometrically finite and have two accidental parabolics: $\frac{1}{0}$ and $\frac{p_{n}}{q_{n}}$. The end invariants for $N_{n}$ are the rational points $\frac{1}{0}$ and $\frac{p_{n}}{q_{n}}$. The definition of the rational numbers $\frac{p_{n}}{q_{n}}$ will be precisely explained in Section 4 . The sequence $\frac{p_{n}}{q_{n}}$ converges to an irrational number $\xi$, defined later.

For the existence of accidental parabolics, the limit set $\Lambda_{n}$ is made by infinite many tangent circles in the complex plane where the tangency points are fixed points of parabolics. We prove that every point in the limit set can be written as an infinite product of the generators $a$ and $b$ and their inverses. Therefore the limit

Received by the editors January 19, 2005.

2000 Mathematics Subject Classification. Primary 30F40; Secondary 57M50.

Key words and phrases. Kleinian groups, limit sets.

(C)2006 American Mathematical Society Reverts to public domain 28 years from publication 
set can be divided into four regions $\Lambda_{a}, \Lambda_{b}, \Lambda_{a^{-1}}$ and $\Lambda_{b^{-1}}$, according to the first letter of the representation as an infinite product.

Among all tangent circles in $\Lambda_{n}$, we choose a chain of tangent circles $C_{n, j}$. These circles separate $\Lambda_{a}$ from the regions $\Lambda_{b}, \Lambda_{a^{-1}}, \Lambda_{b^{-1}}$. The reader can look at Figure 1 and Figure 2 for two examples of the chain. The purple circles represent the circles $C_{n, j}$, the red points are those which can be represented by an infinite word beginning with $b^{-1}$, the green points start with $a^{-1}$, the yellow points start with $b$ and the blue points start with $a$. From Figure1 and Figure2, it is evident that the limit set $\Lambda_{n}$ is very complicated and therefore it is hard to describe. The aim of this work is to analyze it from both an algebraic and geometric point of view. To describe the limit set $\Lambda_{n}$ with an algebraic language, we prove, in the following theorem, that tangency points between consecutive circles in the chain can be described in terms of the Cannon-Thurston maps $\psi_{n}$ for $G_{n}$.

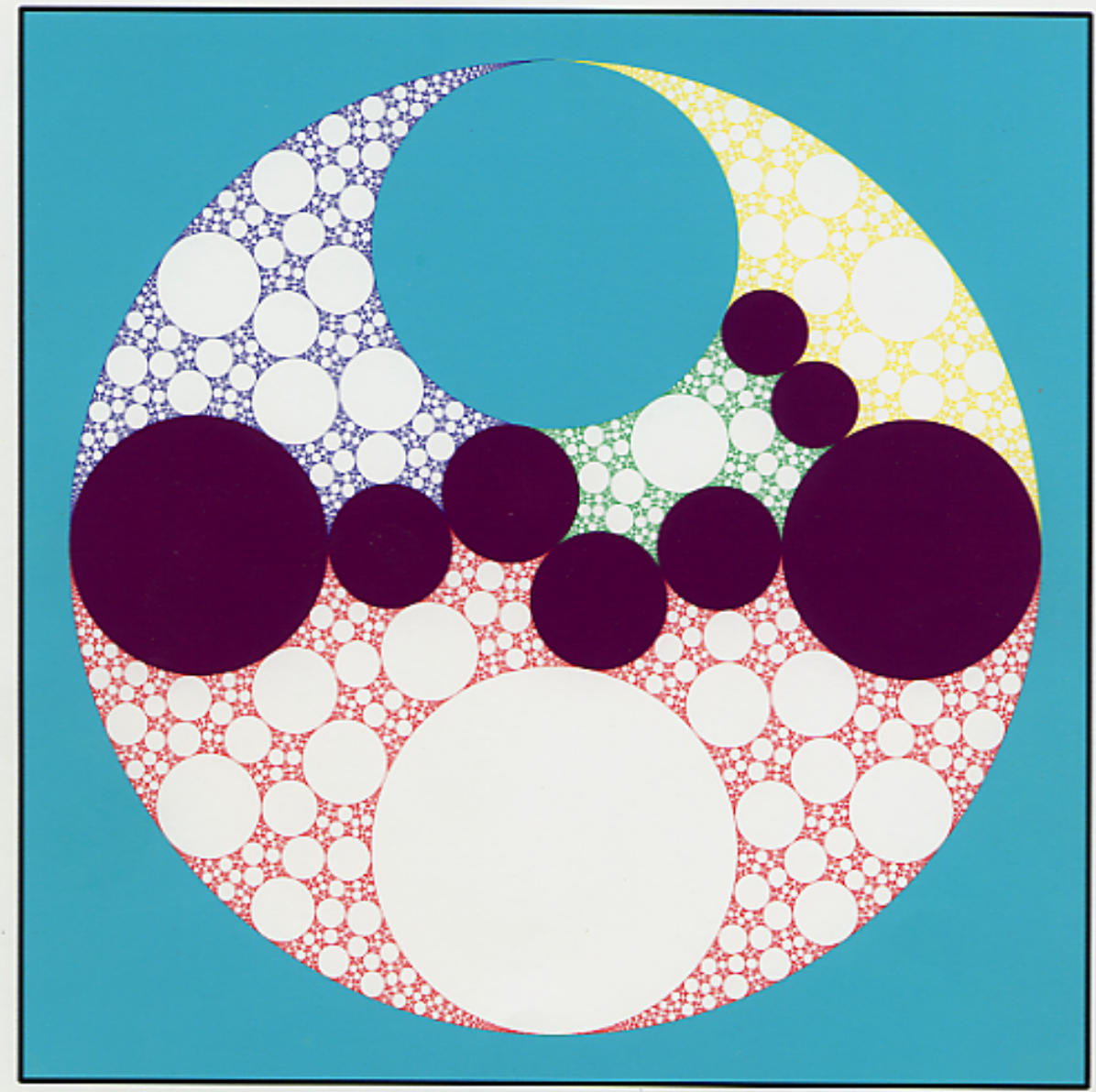

Figure 1. The core chain of circle for $G=\left(\frac{1}{0}, \frac{2}{5}\right)$ 


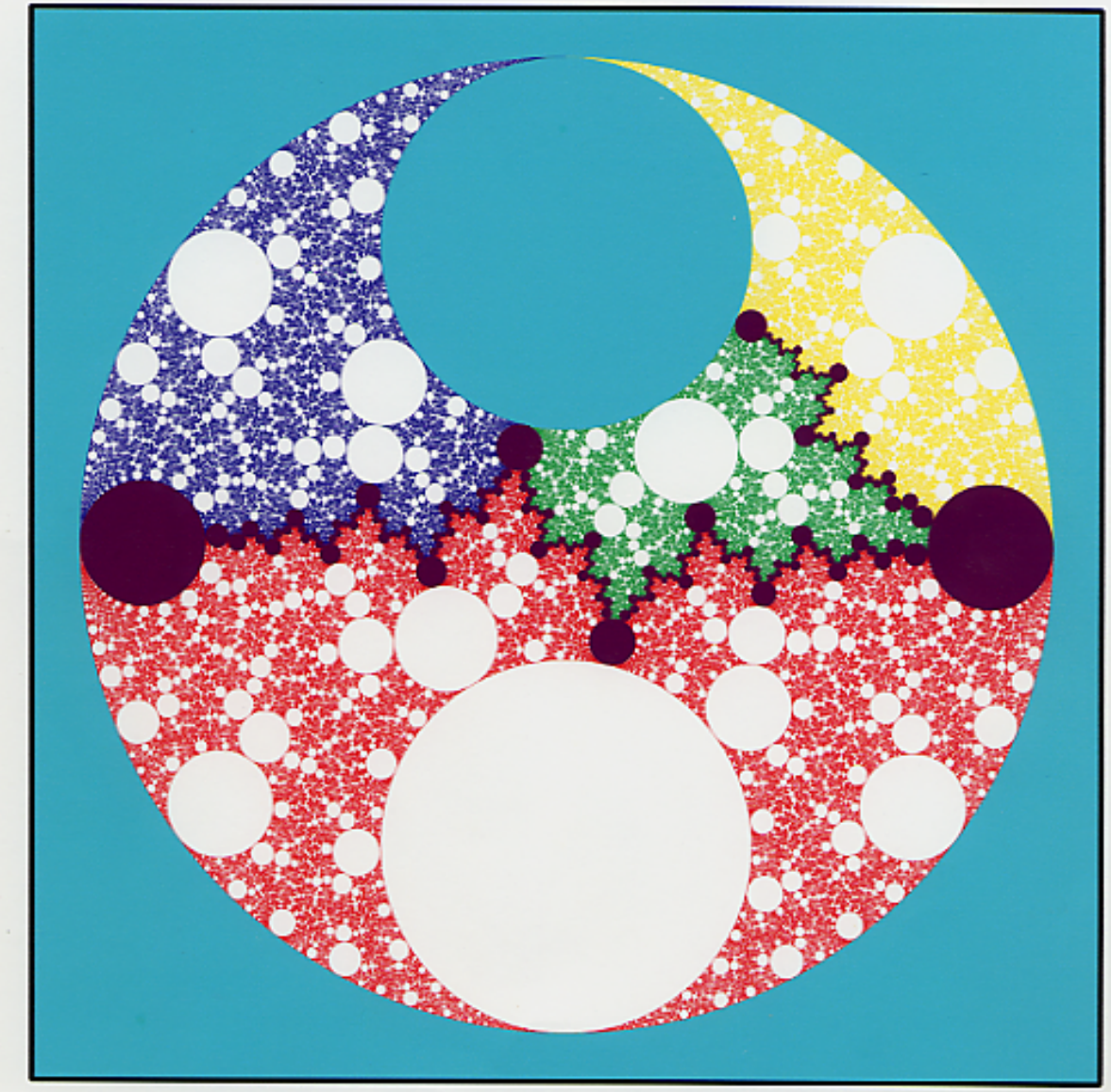

Figure 2. The core chain of circle for $G=\left(\frac{1}{0}, \frac{55}{144}\right)$

Theorem 1.1. The core chain of circles separates the complex plane into the four regions consisting of words starting with $a, b, a^{-1}, b^{-1}$. In particular, the tangency points $z_{i}$ between $C_{n, i}$ and $C_{n, i+1}$ are of the form:

$$
z_{i}=\psi_{n} \circ \varpi\left(\overline{X_{i}}\right)=\psi_{n} \circ \varpi\left(\overline{Y_{i}}\right)
$$

where $\overline{X_{i}}, \overline{Y_{i}} \in \Sigma, e_{1}\left(X_{i}\right)=b^{-1}$ and $e_{1}\left(Y_{i}\right) \neq b^{-1}$, where $\Sigma$ is defined in Section 3.2 and $e_{1}(W)$ is the first letter of the word $W$.

This is a good description of the limit points of $\Lambda_{n}$ in terms of the elements of the group $G_{n}$. Unfortunately, this result does not describe the limit set geometrically. From what we said previously, we know that the limit points are arranged in circles, but we do not know how big these circles are. Moreover, we can't compare different limit sets $\Lambda_{n}$, varying the number $n$. To answer these questions, we prove that the radii of the circles in the circle chain depend only on $n$, the number of elements in the continued fraction expansion of $\frac{p_{n}}{q_{n}}$. First, we prove this result for the first 
circle in the chain, and then we use some symmetries to extend the results for each circle in the chain.

Let $f(x)$ and $g(x)$ be two functions of $x \in \mathbb{R}$ and $a \in \mathbb{R}$. We say that $f$ and $g$ have the same order w.r.t. $a$ (denoted $f \sim_{a} g$ ) if there exists a constant $k \neq 0$ such that $\frac{f}{g} \rightarrow k$ as $x \rightarrow a$. We will omit $a$ when it is clear from the context. Then we prove the following theorem:

Theorem 1.2. The radius $r_{n, 0}$ of $C_{n, 0}$ satisfies $r_{n, 0} \sim \frac{1}{n}$. In particular, $r_{n, 0}$ tends to 0 when $n \rightarrow \infty$.

Corollary 1.3. The radius $r_{n, j}$ of the circle $C_{n, j}$ tends to 0 when $n \rightarrow \infty$ for all $j$.

To prove theorem 1.2, we use the model manifold introduced by Minsky in [22, some results about Margulis tubes that can be found in Section 2.2 and estimates of the lengths of geodesics in the manifold.

An immediate consequence of theorem 1.2 is an application to McShane's identity. This identity was introduced by McShane in [19] and involves the lengths of simple closed curves on a punctured torus. It was extended by Bowditch in [7] to hyperbolic 3-manifolds and it was used by Akiyoshi, Miyachi and Sakuma in 1 for the width of the limit set. Using Jørgensen's normalisation of the once-punctured torus groups, the limit set of the group is contained in a band of a certain height (twice the width). In [1, Akiyoshi, Miyachi and Sakuma proved that the width of the limit set is related to McShane's identity. We prove that this height is proportional to $n$.

Corollary 1.4. Let $\omega\left(\Lambda_{n}\right)$ be the width of the limit set. Then

$$
\omega\left(\Lambda_{n}\right)=\Im \sum_{\gamma \in S_{L}} \frac{1}{1+e^{\lambda(\gamma)}}=-\Im \sum_{\gamma \in S_{R}} \frac{1}{1+e^{\lambda(\gamma)}} \sim n .
$$

Results such as theorem 1.2 and corollary 1.3 are also used in 25] for studying the limit set of the limiting manifold $N_{\infty}$.

At the end of the paper, we analyze the particular case of $n=3$, that is, the manifold $N_{q}$ with end invariants $\frac{1}{0}$ and $\frac{1}{q}$. This case is interesting because the simplicity of the combinatorics allows us to give an estimate about the radius of every circle in the chain.

Theorem 1.5. The radius of $C_{k}$ such that $4<k \leq \frac{q}{2}$ satisfies $r_{k} \sim \frac{1}{e^{V_{1}}}$ where

$$
V_{1}=\frac{4 k}{\sqrt{k^{2}-1}}+\log \left(\frac{\pi^{2}\left(k^{2}-1\right)}{2 c_{4}}\right) .
$$

The constant $c_{4}$ is universal. In particular, the radius of $C_{\frac{q}{2}}$ satisfies $r_{\frac{q}{2}} \sim\left(\frac{4}{q^{2}}\right)$. Moreover, $r_{\frac{q}{m}}$ tends to 0 when $q \rightarrow \infty$.

Moreover, we show that $N_{q}$ is an example of a sequence of manifolds for which the algebraic limit is different from the geometric limit.

The paper is structured as follows.

In Section 2 we give definitions and prove some preliminary facts. We introduce once-punctured torus groups and, in particular, we describe Maskit's embedding. We prove some properties about Margulis tubes of 3-manifolds that we will use in the following part of the paper. 
In Section 3, we define the core chain of circles in the limit set of the manifold. We describe points in the limit set as infinite reduced words in the generators of the group and we relate these matters with Cannon-Thurston maps.

Section 4 is a short description of the limit manifold $N_{\infty}$.

In Section [5] we prove the main theorem of this paper, i.e. the estimates of the radii of the circles and consequently their convergence.

In Section 6. we apply the previous results to relate the number $n$ with McShane's identity and the width of the limit set.

In Section [7, we describe the particular case of $n=3$.

\section{Definitions, NOtATiOn AND PRELIMINARY FACTS}

2.1. Once-punctured torus groups. In this paper, we consider once-punctured torus groups $G$, i.e. free, discrete two generator groups of Möbius transformations such that the commutator of the two generators is parabolic.

The group $G$ can be seen as $\rho\left(\pi_{1}(S)\right)$ where $S$ is a hyperbolic once-punctured torus and

$$
\rho: \pi_{1}(S) \longrightarrow P S L_{2}(\mathbb{C})
$$

is a faithful representation.

Let $N$ be the hyperbolic 3-manifold given by $N=\mathbb{H}^{3} / G$. The loop around the puncture of the torus determines a cusp (which we will call the "main cusp") in $N$ and therefore the representation is type-preserving.

Bonahon [5] proved that $N$ is homeomorphic to the product $S \times(-1,1)$ and it has two ends that we call $e_{+}$and $e_{-}$.

Let $\Lambda \subseteq \hat{\mathbb{C}}$ be the limit set, i.e. the set of accumulation points of the orbit of a point $x \in \mathbb{H}^{3} \cap \hat{\mathbb{C}}$. Let $\Omega=\hat{\mathbb{C}} \backslash \Lambda$ be the ordinary set. The group $G$ acts properly discontinuously on $\Omega$.

From Ahlfors' finiteness theorem, the boundary $\Omega / G$ of $\left(\mathbb{H}^{3} \cup \Omega\right) / G$ is a Riemann surface.

Any component of $\Omega / G$ is reached by going towards one of the two ends, and this divides it into two disjoint pieces $\Omega_{+} / G$ and $\Omega_{-} / G$ (where $\Omega_{+}$and $\Omega_{-}$are the possibly empty corresponding invariant subsets of the ordinary set $\Omega$ ). Since there is a nearest point retraction from $\Omega$ to the boundary of the convex hull $C H(\Lambda)$ of the limit set $\Lambda$, there is a one-to-one correspondence between the components of $\Omega / G$ and the components of the boundary of the convex core $C(N)=C H(\Lambda) / G$. Therefore $\partial C(N)$ is naturally divided into two possibly empty parts $\partial_{+} C(N)$ and $\partial_{-} C(N)$, that are convex pleated surfaces in $N$ with an induced hyperbolic metric.

To classify once-punctured torus groups, we have to understand the two end invariants $\nu_{+}$and $\nu_{-}$that are defined by the two components $\Omega_{+} / G$ and $\Omega_{-} / G$ of $\Omega / G$. In [15], Maskit classified all the possible components of $\Omega / G$ for a general surface. Let us see the classification for the once-punctured torus groups. Let $s$ denote either + or - . There are three possibilities for $\Omega_{s} / G$ :

(1) $\Omega_{s}$ is a topological disk, $\Omega_{s} / G$ and $\partial_{s} C(N)$ are punctured tori. The punctured torus $\Omega_{s} / G$ determines a point $\nu_{s}$ in the Teichmüller space $T(S)$ of $S$. In this case $e_{s}$ is geometrically finite.

(2) $\Omega_{s}$ is an infinite union of disks, $\Omega_{s} / G$ and $\partial_{s} C(N)$ are thrice-punctured spheres and $\partial_{s} C(N)$ is totally geodesic. $\Omega_{s} / G$ is obtained from the corresponding boundary of $S \times(-1,1)$ by deleting a simple closed curve $\gamma_{s}$. 
We define $\nu_{s} \in \hat{\mathbb{Q}}$ as the slope of $\gamma_{s}$. The conjugacy class of $\gamma_{s}$ in $G$ is parabolic. Also, in this case, $e_{s}$ is geometrically finite.

(3) $\Omega_{s}$ is empty. In this case Bonahon [5] and Thurston [27] proved that there is a sequence of simple closed curves $\gamma_{n}$ whose geodesic representatives in $N$ are eventually contained in any neighbourhood of the end $e_{s}$ and whose rational slopes converge to an irrational number $\nu_{s} \in \mathbb{R} \backslash \hat{\mathbb{Q}}$, independent of the sequence $\gamma_{n}$. We say that $\gamma_{n}$ exit the geometrically infinite end, and $\nu_{s}$ is called the ending lamination.

Since the natural boundary of the Teichmüller space $T(S)$ of the punctured torus $S$ is $\hat{\mathbb{R}}$, for each component $\Omega_{s} / G$, in all three cases, we have defined a point in the closure of the Teichmüller space $\overline{T(S)}$.

Therefore the couple $\left(\nu_{-}, \nu_{+}\right)$parameterizes the punctured torus groups and the two elements $\nu_{+}$and $\nu_{-}$are called end invariants.

Let $\Delta$ be the diagonal of the product $\overline{T(S)} \times \overline{T(S)}$. We can notice that the end invariants cannot be the same (otherwise the manifold does not admit a hyperbolic structure).

If both end invariants are rational, i.e. when $\left(\nu_{-}, \nu_{+}\right) \in \hat{\mathbb{Q}} \times \hat{\mathbb{Q}}$, then $G$ is called a double-cusp group.

Now we have all the ingredients for stating the following theorem proved by Minsky in 22].

Theorem 2.1 (Ending lamination theorem). A marked punctured-torus group $\rho$ : $\pi_{1}(S) \longrightarrow P S L_{2}(\mathbb{C})$ is determined by its end invariants $\left(\nu_{-}, \nu_{+}\right) \in(\overline{T(S)} \times \overline{T(S)}) \backslash \Delta$, uniquely up to conjugacy in $P S L_{2}(\mathbb{C})$.

(Marked punctured-torus group means that we keep the representation $\rho$ in mind.) In other words, the map

$$
\rho \mapsto\left(\nu_{-}, \nu_{+}\right)
$$

is injective. It can also be shown to be surjective [22, as a consequence of Bers' simultaneous uniformization theorem [4] and of Thurston's double limit theorem [26].

2.2. The Margulis tubes. In this section we want to describe more precisely the geometry of the Margulis tubes and, in particular, we want to find some estimates for the length of a curve inside the tube depending on the distance between this curve and the core.

The $\epsilon$-thin part of a manifold $N$ (denoted by $N_{\text {thin }}$ ), is the subset of $N$ where the injectivity radius is less then $\epsilon / 2$. The Margulis lemma implies that for each dimension $n$ there is a value of $\epsilon$, known as the Margulis constant, below which the $\epsilon$-thin part of a hyperbolic $n$-manifold is of a standard type. In dimension 3 we denote this constant $\epsilon_{0}$. In this dimension, in the orientable case, every component known as a Margulis tube, is either a tubular neighbourhood of a closed geodesic or a quotient of a horoball by an abelian parabolic group. Let $T_{\epsilon_{0}}(g)$ be the $\epsilon_{0}$-Margulis tube in $N=\mathbb{H}^{3} / G$ for a conjugacy class $g \in G$. With a fixed representation $\rho: \pi_{1}(S) \longrightarrow P S L_{2}(\mathbb{C})$ and a conjugacy class $\alpha \in \pi_{1}(S)$, let $T_{\epsilon_{0}}(\alpha)$ denote $T_{\epsilon_{0}}(\rho(\alpha))$.

Let $r(\alpha)$ be the radius of $T_{\epsilon_{0}}(\alpha)$, that is, the distance from the geodesic core to the boundary of the tube. 
We now prove a proposition that will be very useful in our calculations.

Proposition 2.2. If

$$
l(\alpha)<\frac{\sqrt{3}}{4 \pi}[\log (\sqrt{2}+1)]^{2},
$$

then there exists a constant $k$ such that

$$
r(\alpha) \geq \frac{k}{2} \log \left(\frac{1}{l(\alpha)}\right) .
$$

Proof. From a theorem of Meyerhoff [20], if $l(\alpha)<\frac{\sqrt{3}}{4 \pi}[\log (\sqrt{2}+1)]^{2}$, then there exists an embedded solid tube around $\alpha$ whose radius $r$ satisfies:

$$
\sinh ^{2} r=\frac{1}{2}\left(\frac{\sqrt{1-2 \zeta}}{\zeta}-1\right)
$$

where

$$
\zeta=\cosh \left(\sqrt{\frac{4 \pi l(\alpha)}{\sqrt{3}}}\right)-1 .
$$

The existence of this solid tube is proved by using Jørgensen's inequality. Since $\cosh (x)$ has the following Taylor expansion: $\cosh (x) \sim 1+\frac{x^{2}}{2}$, then

$$
\zeta \sim \frac{c l(\alpha)}{2}
$$

where $c=\frac{4 \pi}{\sqrt{3}}$.

Therefore

$$
\sinh ^{2} r=\frac{1}{2}\left(\frac{2 \sqrt{1-c l(\alpha)}}{c l(\alpha)}-1\right) \sim \frac{\sqrt{1-c l(\alpha)}}{\operatorname{cl}(\alpha)} \sim \frac{1}{l(\alpha)},
$$

as $l(\alpha) \longrightarrow 0$.

Using $\sinh (x)^{-1}=\log \left(x+\sqrt{x^{2}+1}\right)$, we obtain the estimate:

$$
\begin{gathered}
r=\sinh ^{-1}\left(\frac{1}{\sqrt{l(\alpha)}}\right)=\log \left(\frac{1}{\sqrt{l(\alpha)}}+\sqrt{\frac{1}{l(\alpha)}+1}\right) \\
\sim \log \left(\frac{1}{\sqrt{l(\alpha)}}\right)=\frac{1}{2} \log \left(\frac{1}{l(\alpha)}\right) .
\end{gathered}
$$

This implies that there exists a constant $k$ such that

$$
r(\alpha) \geq \frac{k}{2} \log \left(\frac{1}{l(\alpha)}\right) .
$$

Proposition 2.3. Let $\lambda(\alpha)=l(\alpha)+i \theta(\alpha)$ be the complex translation length of $\alpha$. Let $L(h)$ be the length of a curve homotopic to $\alpha$ at distance $h$ from the core of the Margulis tube.

Then $L(h)$ satisfies the following equation:

$$
\sinh ^{2} h=\frac{\cosh (L(h))-\cosh (l)}{\cosh (l)-\cos (\theta)} .
$$




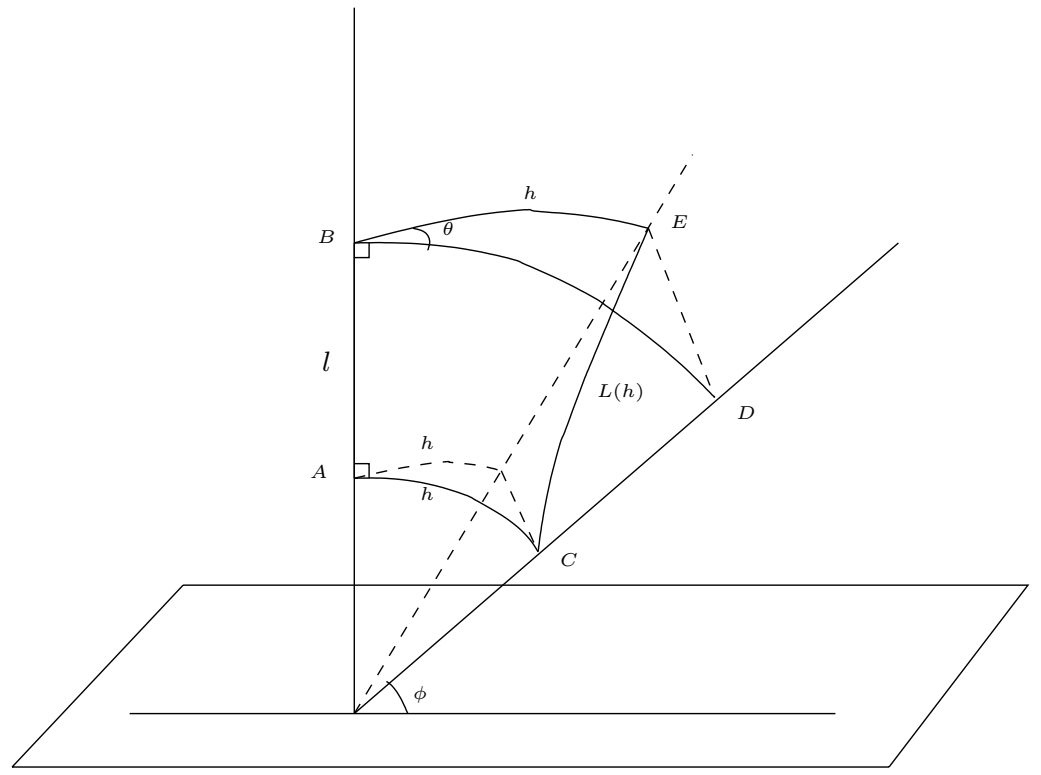

FiguRE 3. The Margulis tube around a pivot

Proof. In Figure 3 we describe the Margulis tube $T_{\epsilon_{0}}(\alpha)$.

The imaginary axis represents the axis of $\alpha$. Following the labels of the picture, the point $A$ is sent by $\alpha$ to $B$ and therefore $d(A, B)=l(\alpha)$ is the translation length of $\alpha$. The three geodesics passing through the points $A, C$, the points $B, D$ and the points $B, E$ are perpendicular to the axis of $\alpha$. It is easy to check that $d(A, C)=d(B, D)=d(B, E)=h$, since the points $C, D, E$ belong to lines passing through the origin with the same angle $\phi$ from the complex plane.

We can calculate $L(h)$ introducing coordinates. Let the points $A, B, C, D$, and $E$ be as in the picture. We can suppose that $A=j$ and $B=b j$ (using quaternions). Since $d(A, B)=\log (b)=l$, then $B=e^{l} j$.

In coordinates we can write $C=c_{1}+c_{2} i+c_{3} j$ and $E=e_{1}+e_{2} i+e_{3} j$. Simple geometry shows that $C=\cos \phi+\sin \phi j$ and $E=e^{l}(\cos \phi \cos \theta+\cos \phi \sin \theta i+\sin \phi j)$. Moreover, from the Angle of Parallelism in hyperbolic geometry, $\sin \phi=\frac{1}{\cosh h}$ (see [3]).

Using a well-known formula for the hyperbolic distance between two points (for more details see Beardon [3]),

$$
\begin{gathered}
\cosh (d(C, E))=\frac{\left|c_{1}+c_{2} i-e_{1}-e_{2} i\right|^{2}+c_{3}^{2}+e_{3}^{2}}{2 c_{3} e_{3}} \\
=\frac{\left|a \cos \phi-a e^{l} \cos \phi \cos \theta-a e^{l} \cos \phi \sin \theta i\right|^{2}+a^{2} \sin ^{2} \phi+a^{2} e^{2 l} \sin ^{2} \phi}{2 a^{2} e^{l} \sin ^{2} \phi} \\
=\frac{1+e^{2 l}-2 e^{l} \cos ^{2} \phi \cos \theta}{2 e^{l} \sin ^{2} \phi}=\frac{1+e^{2 l}}{2 e^{l}} \cosh ^{2}(h)-\cot ^{2} \phi \cos \theta .
\end{gathered}
$$


Using that $\cot ^{2} \phi=\cosh ^{2} h-1$ and that $\cosh l=\frac{e^{2 l}+1}{2 e^{l}}$, we obtain the following equation:

$$
\begin{aligned}
\cosh (L(h)) & =\cosh (d(C, E))=\cosh (l) \cosh ^{2}(h)-\cos \theta\left(\cosh ^{2}(h)-1\right) \\
& =\cosh (l)+\cosh (l) \sinh ^{2}(h)-\cos \theta \sinh ^{2}(h),
\end{aligned}
$$

and therefore

$$
\sinh ^{2} h=\frac{\cosh (L(h))-\cosh (l)}{\cosh (l)-\cos (\theta)} .
$$

2.3. Convergence of groups. In this section we will explain the difference between geometric limit and algebraic limit for once-punctured torus groups acting on $\mathbb{H}^{3}$.

Let $H(S)$ denote the quotient $H(S)=D(S) / P S L_{2}(\mathbb{C})$, acting by conjugation, where $D(S)$ is the space of discrete, faithful representations. On $H(S)$ we can define the following "natural" algebraic topology:

$$
\left[\rho_{n}\right] \stackrel{\text { alg }}{\longrightarrow}[\rho]
$$

if there are representatives of each equivalence class such that

$$
\rho_{n}(g) \longrightarrow \rho(g),
$$

for each $g \in \pi_{1}(S)$. Then $A H(S)$ is $H(S)$ endowed with the algebraic topology.

We now give a notion of geometric convergence. We say that a sequence of manifolds with baseframes $\left(N_{i}, \omega_{i}\right)$ converges to a manifold with baseframe $(N, \omega)$, denoting

$$
\left(N_{i}, \omega_{i}\right) \stackrel{g e o}{\longrightarrow}(N, \omega),
$$

if and only if for each compact submanifold $K \subseteq N$ containing the baseframe $\omega$, there are smooth embeddings $f_{i}: K \longrightarrow N_{i}$, defined for all $i$ sufficiently large, such that $f_{i}$ sends $\omega$ to $\omega_{i}$ and $f_{i}$ tends to an isometry in the $C^{\infty}$-topology.

The algebraic convergence and the geometric convergence are not equivalent and the reader can see [17, [10, 27, and [12] for some examples. Nevertheless, there are many theorems (see [8], 2] ) in which, with some appropriate conditions, the algebraic convergence implies the geometric convergence. One such condition is that the limit manifold has no accidental parabolics.

Definition 2.4. We say that

$$
\left(N_{i}, \omega_{i}\right) \stackrel{\text { strongly }}{\longrightarrow}(N, \omega)
$$

if the convergence is algebraic and geometric.

2.4. Maskit's embedding. If $(\alpha, \beta)$ is a marking of $S$, and if $a=\rho(\alpha)$ and $b=\rho(\beta)$, then the commutator $K=b^{-1} a^{-1} b a$ is parabolic.

Among all once-punctured torus groups, we consider the ones where one generator (we can suppose $a$ ) is parabolic. The group depends only on one complex parameter $\operatorname{tr}(b)$. This group is called Maskit's embedding of the once-punctured torus for the name of the mathematician who first studied these groups (see [16]).

We can normalize the group (see [28]) such that

$$
a=\left(\begin{array}{ll}
1 & 2 \\
0 & 1
\end{array}\right)
$$


and

$$
b_{\mu}=\left(\begin{array}{cc}
-i \mu & -i \\
-i & 0
\end{array}\right)
$$

Therefore $a$ is the translation defined by the equation $a(z)=z+2$, that is parabolic with $\infty$ as a fixed point and $b_{\mu}$ is the Möbius transformation $b_{\mu}(z)=\frac{\mu z+1}{z}$ and its trace satisfies $\operatorname{tr}\left(b_{\mu}\right)=-i \mu$.

The commutator $K=b^{-1} a^{-1} b a$ is

$$
K=\left(\begin{array}{cc}
1 & 2 \\
-2 & -3
\end{array}\right)
$$

its trace is -2 and $K$ acts on the complex plane as $K(z)=\frac{z+2}{-2 z-3}$ with fixed point -1 .

Sometimes we denote $G(\mu)$ as the group $G$, since it only depends on the complex parameter $\mu$.

From the point of view of the end invariants and Teichmüller parameters, among all discrete torsion-free groups $G(\mu)$, we want to consider those with end invariants $\nu^{-}=\frac{1}{0}$ and $\nu^{+} \in \mathbb{H}^{2}$. The Maskit embedding is the induced map:

$$
\begin{gathered}
T(S) \\
\nu^{+} \longmapsto \mathbb{C}, \\
\quad \mu .
\end{gathered}
$$

Maskit proved [16 that this map is a holomorphic bijection.

From what we have seen in Section $2, \Omega_{-} / G$ is a thrice-punctured sphere.

Now we describe the relation between the elements of a group $G$ with generators $a$ and $b$ and the curves on the punctured torus $S=\mathbb{H}^{2} / G$.

Definition 2.5. Any element $g \in G$ that corresponds to a non-trivial, nonperipheral simple closed curve on the once-punctured torus is called generator. We say that two generators $g$ and $h$ are neighbours if the corresponding simple closed curves have intersection number 1 .

Any pair of neighbours are defined up to conjugation of both elements, interchanging the order and taking inverses of either element (see [24.) Any pair of neighbours $g$ and $h$ generates $G$ [24].

Definition 2.6. Given a pair of generators $(g, h)$, a Nielsen move sends the couple $(g, h)$ to one of $(g, g h),\left(g, g^{-1} h\right),(g h, h)$ or $\left(g h^{-1}, h\right)$.

Topologically these Nielsen moves correspond to performing a Dehn twist about the curve corresponding to either $g$ or $h$.

Definition 2.7. If $g$ and $h$ are neighbours, the set $(g, h, g h)$ is called a generator triple.

The generator triple is defined up to interchanging the order, taking the inverse and conjugation.

In what follows, we are going to identify the Teichmüller space $T(S)$ of the punctured torus $S$ with the hyperbolic plane $\mathbb{H}^{2}$ and we can see its compactification as the circle $\hat{\mathbb{R}}=\mathbb{R} \cup \infty$. In this view, the set of (non-peripheral) simple closed curves on $S$ is naturally identified with $\hat{\mathbb{Q}}$. Let us describe this identification.

Fixing a marking $(\alpha, \beta)$ of the torus $S$, we can associate to this marking the lattice generated by 1 and $z \in \mathbb{H}^{2}$, whose quotient is a torus with induced conformal structure $z$. An orientation-preserving identification of the fixed torus $S$ with this 
torus is determined by taking the curve $\alpha$ to the image of $[0,1]$ and $\beta$ to the image of $[0, z]$. The position of the puncture is irrelevant since the torus has a transitive family of conformal automorphism. Any free homotopy class of unoriented simple non-peripheral curves on $S$ is a combination $p \alpha+q \beta$ of the two generators $\alpha$ and $\beta$. Therefore we associate for each element in $H_{1}(S)$ its slope $-\frac{p}{q} \in \hat{\mathbb{Q}}$ and we associate for each generator $g$ its slope $-\frac{p}{q}$.

Using projective measured laminations $P M L(S)$ on the torus, Thurston 27] generalised the correspondence between simple closed curves and rational points, since he proved that $P M L(S)$ is homeomorphic to $S^{1} \simeq \mathbb{R} \cup \infty$. In this interpretation, laminations with infinite leaves correspond to irrational points.

Two generators are neighbours if the corresponding rational numbers $\frac{p}{q}$ and $\frac{r}{s}$ satisfy $p s-q r= \pm 1$.

Since the generators correspond to rational points and because of the relationship between neighbours and slope, the Farey graph is a good geometric model for this set (the Farey graph is a 1-skeleton where the vertices correspond to generators and two vertices are connected by an edge if they are neighbours).

Now we want to explicitly describe the elements of the group in terms of the slopes of the corresponding curves on $S$.

Using induction, we define the words $W_{\frac{p}{q}}$ corresponding to the curve of slope $\frac{p}{q}$ :

$$
W_{\frac{n}{1}}=a^{-n} b, W_{\frac{0}{1}}=b \quad \text { and } \quad W_{\frac{1}{1}}=a^{-1} b .
$$

If $\left(\frac{p}{q}, \frac{r}{s}\right)$ are Farey neighbours with $\frac{p}{q}<\frac{r}{s}$, then

$$
W_{\frac{p+r}{q+s}}=W_{\frac{r}{s}} W_{\frac{p}{q}} .
$$

The following two lemmas are proved in [14].

Lemma 2.8. For $0 \leq \frac{p}{q} \leq 1$ we have $W_{\frac{p}{q}}=a^{-1} b^{n_{1}} a^{-1} b^{n_{2}} \ldots a^{-1} b^{n_{p}}$ with $\sum_{i=1}^{p} n_{i}$ $=q$ and $\left|n_{i}-n_{j}\right| \leq 1$ where $1 \leq i, j \leq p$.

Lemma 2.9. For each pair of Farey neighbours $\left(\frac{p}{q}, \frac{r}{s}\right)$ with $0 \leq \frac{p}{q}<\frac{r}{s} \leq 1$, $\left(W_{\frac{p}{q}}, W_{\frac{r}{s}}\right)$ generates the group $G$, and

$$
K=\left[b^{-1}, a^{-1}\right]=\left[W_{\frac{r}{s}}^{-1}, W_{\frac{p}{q}}\right] .
$$

The following proposition about the trace of the $\frac{p}{q}$-word is proved in [14 by induction.

Proposition 2.10. The trace of $W_{\frac{p}{q}}$ is a polynomial of the form:

$$
\operatorname{tr}\left(W_{\frac{p}{q}}\right)=(-i)^{q}\left(\mu^{q}-2 p \mu^{q-1}+b_{q-2} \mu^{q-2}+\cdots+b_{0}\right), b_{i} \in \mathbb{Z} .
$$

Let us consider $D$, the space of parametrisation for $G(\mu)$. Many studies and many approximations with the computer have been made to find the shape of $D$ and of its boundary $\partial D$.

An element $\mu$ outside $D$ corresponds to a non-discrete or non-free group and $\mu$ in the interior of $D$ corresponds to a single-cusp group, i.e. a group for which $\Omega_{+} / G$ is a once-punctured torus and $\Omega_{-} / G$ is a thrice-punctured sphere. On the other hand, a point $\mu \in \partial D$ corresponds to a double-cusp group or a singly degenerate group. Let us see this in more detail in the following two theorems. The first theorem was Maskit's conjecture: The existence of $\mu\left(\frac{p}{q}\right)$ is proved by Maskit in [16] whereas 
the uniqueness is proved in [13. The second theorem is a strong version of the Bers conjecture for Maskit's embedding of the once-punctured torus and it is proved by Minsky in 22].

Theorem 2.11. For each $\frac{p}{q} \in \mathbb{Q}$, there exists a unique $\mu\left(\frac{p}{q}\right) \in \partial D$ such that $\operatorname{tr}\left(W_{\frac{p}{q}}\right)= \pm 2$ in $G\left(\mu\left(\frac{p}{q}\right)\right)$.

Moreover, in $G\left(\mu\left(\frac{p}{q}\right)\right), \operatorname{tr}\left(W_{\frac{r}{s}}\right) \neq \pm 2$ for all rationals $\frac{r}{s} \neq \frac{p}{q}$.

Theorem 2.12. The mapping,

$$
\frac{p}{q} \longmapsto \mu\left(\frac{p}{q}\right)
$$

extends to a homeomorphism of $\mathbb{R}$ onto the boundary of $D$ :

$$
\begin{gathered}
\mathbb{R} \longrightarrow \partial D, \\
r \longmapsto \mu(r) .
\end{gathered}
$$

Each point $\mu\left(\frac{p}{q}\right) \in \partial D$ (given in Theorem 2.11) corresponds to a double-cusp group $G_{\frac{p}{q}}$ with end invariants $\left(\frac{1}{0}, \frac{p}{q}\right)$, that is, a group in which $W_{\frac{p}{q}}$ is accidentally parabolic. Then $\Omega_{+} / G_{\frac{p}{q}}$ is a thrice-punctured sphere and $\Omega_{+}$is a countable union of disks, tangent at the fixed points of the new accidental parabolics.

When $\xi \in \mathbb{R} \backslash \mathbb{Q}$, then $\mu(\xi)$ corresponds to a singly degenerate group with end invariants $\left(\frac{1}{0}, \xi\right)$.

Theorem 2.12 also proves that cusps are dense in $\partial D$.

To understand the shape of $D$ and $\partial D$, it is useful to see some symmetries (whose proof can be found in [28]):

Proposition 2.13. The space $D$ of parametrisation for $G(\mu)$ satisfies the following properties:

(1) $\partial D \subset\{\mu \in \mathbb{C}: 1 \leq \Im(\mu) \leq 2\}$.

(2) $\mu\left(\frac{0}{1}\right)=2 i$.

(3) There is a reflectional symmetry in the imaginary axis $\Re(\mu)=0$.

(4) $D$ is invariant under the translation by 2: If $\mu \in D$, then $\mu+2 \in D$.

From the previous proposition, it is enough to work in $[0,1]$.

\section{Description of the core Chain of Circles $C_{j}$}

In this section we define and describe the main object that we will study in the following, that is, the core chain of circles $C_{j}$.

3.1. The core chain of circles. We remind the reader that in $G_{\frac{p}{q}}=G\left(\mu\left(\frac{p}{q}\right)\right)$, the invariant subset $\Omega_{+}$of the ordinary set is an infinite union of tangent disks.

In this section we define the circle chain $C_{j}$, that is, a subset of the limit set for the cusp-group $G_{\frac{p}{q}}$ (the once-punctured torus group in which $a$ and $W_{\frac{p}{q}}$ are parabolic).

First we can notice that from lemma 2.9, we have that

$$
\operatorname{tr}\left(K W_{\frac{p}{q}}\right)=\operatorname{tr}\left(W_{\frac{p}{q}}\right) .
$$


Since $W_{\frac{p}{q}}$ and $K$ are parabolic, then the subgroup $\Gamma_{0}=\left\langle W_{\frac{p}{q}}, K\right\rangle$, generated by $W_{\frac{p}{q}}$ and $K$, is Fuchsian (because the traces of generators and of generators product are real). Therefore $\Gamma_{0}$ has an invariant circle $C_{0}=\Lambda\left(\Gamma_{0}\right)$.

We will define the circle chain $C_{j}$ as the images of the circle $C_{0}$ by some elements in the group $G \frac{p}{q}$.

Definition 3.1. A $\frac{p}{q}$-circle chain is a sequence $\left\{C_{j}\right\}_{j \in \mathbb{Z}}$ of tangent circles in $\mathbb{C}$ with the following properties:

(1) $C_{0}$ and $C_{q}$ are tangent to $\mathbb{R}$ at -1 and 1 , respectively.

(2) $W_{\frac{p}{q}}\left(C_{0}\right)=C_{0}$.

(3) $C_{r+p}=b\left(C_{r}\right)$ for $0 \leq r<q(b$ pushes forward by $p)$.

(4) $C_{r+q}=a\left(C_{r}\right) \forall r \in \mathbb{Z}$ (a pushes forward by $q$ ).

The existence of the chain is proved in 28 .

There is an alternative inductive procedure (see [14]) to define the circle chain, given the rational number $\frac{p}{q}$ that we describe in what follows.

For $0 \leq r<q$, let $n_{r}=r p \bmod q$ for $0 \leq n_{r}<q$ and set $n_{q}=q$.

For $0 \leq i \leq q$, define words $E_{i}$ by

$$
\begin{gathered}
E_{0}=i d, \\
E_{n_{r+1}}=b E_{n_{r}} \quad \text { if } 0 \leq p+n_{r}<q, \\
E_{n_{r+1}}=a^{-1} b E_{n_{r}} \quad \text { if } p+n_{r} \geq q .
\end{gathered}
$$

Now define $C_{i}=E_{i}\left(C_{0}\right)$ for $1 \leq i<q$. If $k \in \mathbb{Z}$ and $k=r q+s$ with $0 \leq s<q$, define $C_{k}=a^{r} C_{s}$. In particular, for $k=-1, C_{-1}=a^{-1} C_{q-1}$ and $E_{-1}=a^{-1} E_{q-1}$.

For the proof of the following lemma, one can see [14].

Lemma 3.2. Suppose that $W_{\frac{p}{q}}=W_{\frac{c}{d}} W_{\frac{a}{b}}$. Then $E_{1}=W_{\frac{a}{b}}, E_{-1}=W_{\frac{c}{d}}$ and $E_{q}=W_{\frac{p}{q}}$.

After defining the chain, we see how the elements of the group act on the circles (whose proof can be found in [14 and [28]):

\section{Proposition 3.3.}

(1) $K\left(C_{0}\right)=C_{0}, K(-1)=-1, K(\hat{\mathbb{R}})=\hat{\mathbb{R}}, K$ acts on the boundary of $C_{0}$ in a counterclockwise sense.

(2) $W_{\frac{p}{q}}\left(C_{0}\right)=C_{0}, W_{\frac{p}{q}}\left(C_{1}\right)=C_{1}, W_{\frac{p}{q}}\left(z_{0}\right)=z_{0}$ where $z_{0}=C_{0} \cup C_{1}$.

(3) $C_{i}$ is the invariant circle of the Fuchsian group $E_{i}\left\langle W_{\frac{p}{q}}, K\right\rangle E_{i}^{-1}$.

Since $C_{0}$ is the invariant circle of the Fuchsian group $\left\langle W_{\frac{p}{q}}, K\right\rangle$ and for (3) above, then $\Omega_{+}$is the union of the images, by the elements of the group $G_{\frac{p}{q}}$, of the open disks bounded by the circle chain (see [28]).

For the proof of the next theorem, the reader can see [28].

Theorem 3.4. For each non-negative rational number $\frac{p}{q} \leq 1$ (in lowest terms), the circles $C_{j}, 0 \leq j \leq q-1$, satisfy the following properties:

(1) All the closed disks bounded by the circles $C_{j}$ are mutually disjoint with the following exception: For any integer $j, C_{j} \cap C_{j+1}$ consists of a single point $z_{j}$. 
(2) All the circles are strictly contained in $\mathbb{H}_{U}$ (the upper half plane) with the exception of the circles $C_{q l}$, for all integers $l$, which are contained in $\overline{\mathbb{H}_{U}}$ and tangent to $\hat{\mathbb{R}}$ at $2 l-1$.

(3) There are no limit points inside the open disks bounded by the circles $C_{j}$.

3.2. Limit set as infinite words. Since the core chain of circles is made by limit points, we now want to describe points in the limit set as infinite products of the generators of the group and their inverses. More precisely, we want to find a surjective map from the set of infinite reduced words to the limit set $\Lambda\left(G_{\frac{p}{q}}\right)$, and we want to see which are the words that correspond to the same limit point.

In Section 2, we defined the limit set as the set of accumulation points of the orbit of a base point $x$. The limit set is invariant under the group and the definition does not depend on $x$.

Let $\Sigma$ be the set of infinite reduced words in the generators

$$
\Gamma_{0}=\left\{a, b, a^{-1}, b^{-1}\right\},
$$

where a word is reduced if it does not contain canceling sequences like $a a^{-1}$ or $b b^{-1}$.

Let $\Sigma_{F}$ be the set of finite reduced words in $\Gamma_{0}$.

Let us fix a Fuchsian group $G_{0}$ for the once-punctured torus, $G_{0}=\rho_{0}\left(\pi_{1}(S)\right)$.

Let $\mathcal{R}$ be a fundamental domain in the Poincaré disk, containing 0 , with four ideal vertices and four sides $\gamma_{a}, \gamma_{b}, \gamma_{a^{-1}}, \gamma_{b^{-1}}$. The four circular arcs are perpendicular to the unit circle $S^{1}$ and $a, b$ satisfy:

$$
\begin{aligned}
& a\left(\operatorname{ext}\left(\gamma_{a}\right)\right)=\operatorname{int}\left(\gamma_{a^{-1}}\right), \\
& b\left(\operatorname{ext}\left(\gamma_{b}\right)\right)=\operatorname{int}\left(\gamma_{b^{-1}}\right),
\end{aligned}
$$

where $\operatorname{int}\left(\gamma_{g}\right)$ is the region in the Poincare disk, that is, inside the circle which contains the arc $\gamma_{g}$ and $\operatorname{ext}\left(\gamma_{g}\right)$ is the region in the Poincaré disk, that is, outside the circle which contains the arc $\gamma_{g}$.

Let $\mathcal{G}\left(G_{0}\right)$ be the Cayley graph of $G_{0}$. This can be realized in the Poincaré disk as follows: The vertices are points $g(0)$ (labeled $g$ ) with $g \in G_{0}$, and the edges are directed lines joining vertices $g(0), g^{\prime}(0)$ for which $g^{-1} g^{\prime}$ is one of the generators (see Figure 4).

If we consider the Poincaré disk with the Cayley graph $\mathcal{G}\left(G_{0}\right)$ and the fundamental region of $G_{0}$ and its images, we can notice that the arc $\gamma_{g}$ intersects the edge joining $e_{1} \ldots e_{n-1}(0)$ with $e_{1} \ldots e_{n}(0)$ where $g=\left(e_{1} \ldots e_{n}\right)^{-1}$.

There exists a bijection $\varpi_{F}$ from the finite reduced words $\Sigma_{F}$ to the image of 0 by the elements of $G_{0}$, defined as follows:

$$
\begin{aligned}
& \varpi_{F}: \quad \Sigma_{F} \quad \longrightarrow \quad G_{0}(0), \\
& \left(e_{1} \ldots e_{n}\right) \longmapsto e_{1} \ldots e_{n}(0) \text {. }
\end{aligned}
$$

This factors through a bijection $\Sigma_{F} \longrightarrow \pi_{1}(S)$.

It is possible to extend $\varpi_{F}$ to the boundary: There exists a map

$$
\begin{array}{ccc}
\varpi: & \Sigma & \longrightarrow \Lambda\left(G_{0}\right), \\
\left(e_{1} \ldots e_{n} \ldots\right) & \longmapsto \varpi(e),
\end{array}
$$

where $\varpi(e)$ is the accumulation point of $0, e_{1}(0), e_{1} e_{2}(0), \ldots, e_{1} e_{2} \ldots e_{n}(0), \ldots$.

Let us denote by $\bar{g}$, the infinite word $g g g g g \ldots$ in $\Sigma$. If $g$ is parabolic, then the attracting fixed point coincides with the repelling fixed point and therefore

$$
\bar{g}=\overline{g^{-1}} \text {. }
$$




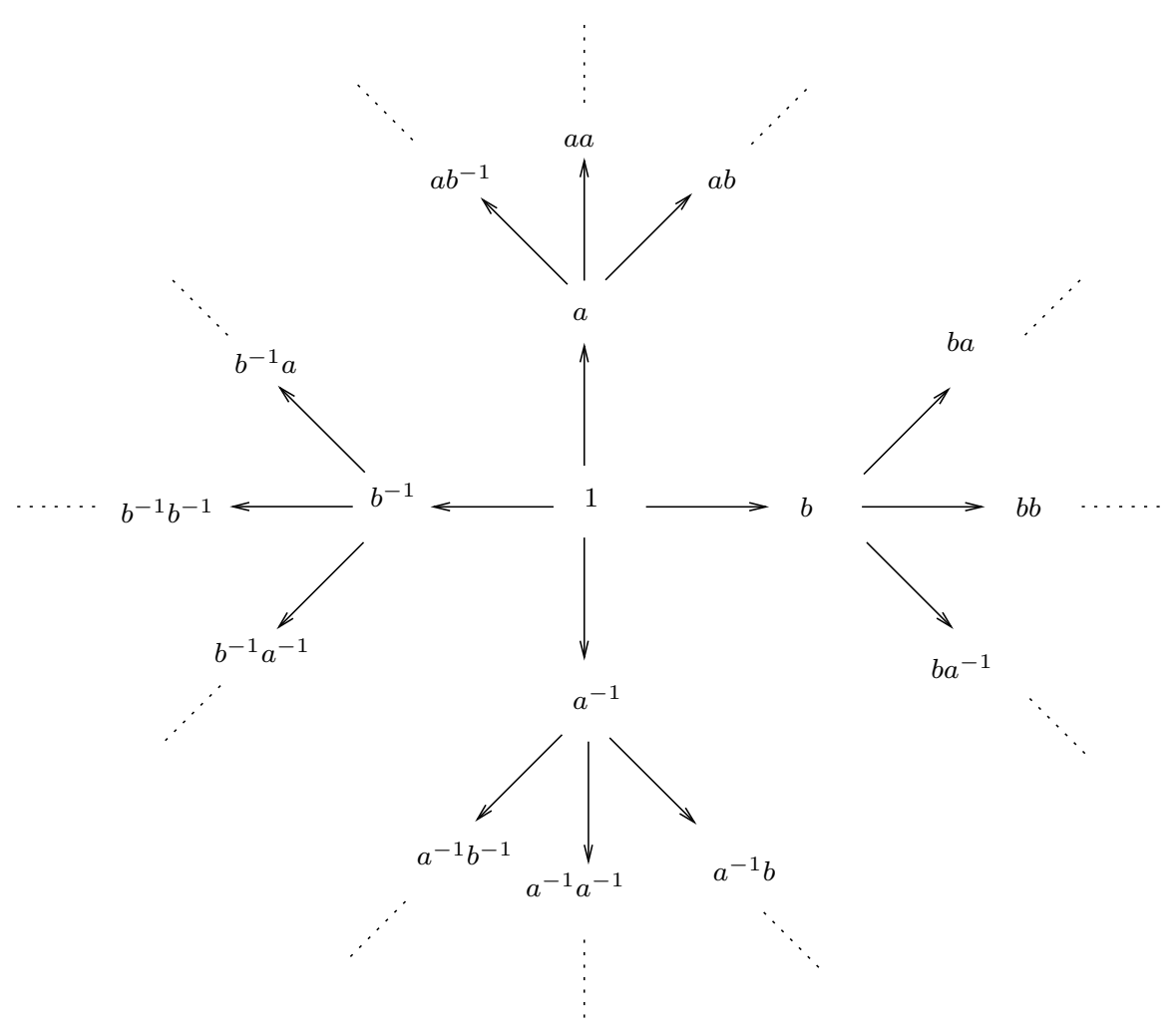

FiguRE 4. The graph for the group with generators $a$ and $b$

Moreover, $\varpi$ is surjective and not injective because the commutator of the two generators is parabolic. The points $x$ in $\Lambda\left(G_{0}\right)$ that are images of two elements in $\Sigma$ are the fixed points of the commutator, and all its conjugates. For more details, the reader can look at 25$]$.

3.3. Cannon-Thurston maps. Now we see how to construct a map $\psi_{\frac{p}{q}}$ from the limit set of a fixed Fuchsian group, to the limit set of the group $G_{\frac{p}{q}}$, that is, the group in which $a$ and $W_{\frac{p}{q}}$ are accidentally parabolic. We will see that this construction can be done in a more general setting (for any group $G$ ) and the map $\psi$ is called the Cannon-Thurston map.

Let us fix a Fuchsian representation $\rho_{0}$ for the once-punctured torus. Let us denote the image of $\pi_{1}(S)$ by $\rho_{0}$ by $G_{0}$.

Definition 3.5. Given a group $G$ with representation

$$
\rho: \pi_{1}(S) \longrightarrow G \subset P S L_{2}(\mathbb{C}),
$$

we say that a map $\psi$ is a Cannon-Thurston map for $(G, \rho)$ if $\psi$ is a continuous map

$$
\psi: \Lambda\left(G_{0}\right) \longrightarrow \Lambda(G)
$$

which satisfies the following equivariance:

$$
\rho(g) \circ \psi(z)=\psi \circ \rho_{0}(g)(z) \forall g \in \pi_{1}(S) \text { and } \forall z \in S^{1} .
$$


The following lemma is proved by McMullen in [18] and asserts that the CannonThurston map for once-punctured torus groups exists and it is unique.

Lemma 3.6. Let $G$ be a once-punctured torus group, with a discrete faithful representation $\rho: G \longrightarrow P S L_{2}(\mathbb{C})$.

Then there exists a Cannon-Thurston map for $(G, \rho)$. Moreover, if two continuous maps $\psi_{1}$ and $\psi_{2}$ satisfies the equivariance (11), then

$$
\psi_{1}=\psi_{2} .
$$

The existence of Cannon-Thurston maps has been proven by many authors ([6], 9], and [23]) for various classes of Kleinian groups.

Let us consider the group $G_{\frac{p}{q}}$.

Let $\psi_{\frac{p}{q}}$ be the Cannon-Thurston map for $G_{\frac{p}{q}}$ :

$$
\psi_{\frac{p}{q}}: \Lambda\left(G_{0}\right) \longrightarrow \Lambda\left(G_{\frac{p}{q}}\right) .
$$

Let us consider the set $\lambda_{\frac{p}{q}}$ of leaves whose endpoints are fixed points of $W_{\frac{p}{q}}$ and its conjugates (then $\lambda_{\frac{p}{q}}$ corresponds to the geodesic in the once-punctured torus with slope $\left.\frac{p}{q}\right)$.

Since, in the group $G_{\frac{p}{q}}$, the element $W_{\frac{p}{q}}$ is an accidental parabolic, then the map

$$
\psi_{\frac{p}{q}}: \Lambda\left(G_{0}\right) \longrightarrow \Lambda\left(G_{\frac{p}{q}}\right)
$$

collapses the endpoints of the leaves of $\lambda \frac{p}{q}$.

For the same reason, since $a$ is accidentally parabolic, the map $\psi_{\frac{p}{q}}$ collapses the fixed points of $a$ and all its conjugates. Floyd, in [9], proved that there is no more collapsing.

Let us denote by $\varpi \frac{p}{q}$ the composition map between $\varpi$ and $\psi_{\frac{p}{q}}$ :

$$
\varpi \frac{p}{q}=\psi_{\frac{p}{q}} \circ \varpi: \Sigma \longrightarrow \Lambda\left(G_{\frac{p}{q}}\right) .
$$

This map represents the relation between the infinite reduced words and the limit set of $G \frac{p}{q}$.

Now we want to characterise the tangency points between circles in the core chain.

By definition, the first tangency point $z_{0}$ between $C_{0}$ and $C_{1}$ is the fixed point of $W_{\frac{p}{q}}$ because $W_{\frac{p}{q}}\left(C_{0}\right)=C_{0}$ and $W_{\frac{p}{q}}$ is parabolic. Therefore,

$$
z_{0}=\varpi \frac{p}{q}\left(\overline{W_{\frac{p}{q}}}\right)=\varpi \frac{p}{q}\left(\overline{W_{\frac{p}{q}}^{-1}}\right) .
$$

Since $C_{i}=E_{i}\left(C_{0}\right)$, then the tangency point $z_{i}$ between $C_{i}$ and $C_{i+1}$ is the fixed point of $E_{i} W_{\frac{p}{q}} E_{i}^{-1}$. Therefore,

$$
z_{i}=\varpi \frac{p}{q}\left(\overline{E_{i} W_{\frac{p}{q}}}\right)=\varpi \frac{p}{q}\left(\overline{E_{i} W_{\frac{p}{q}}^{-1}}\right) .
$$

From lemma 2.8, the word $W_{\frac{p}{q}}$ has the form:

$$
W_{\frac{p}{q}}=a^{-1} b^{n_{1}} a^{-1} b^{n_{2}} \ldots a^{-1} b^{n_{p}} .
$$

Therefore $z_{0}$ has two different representations in $\Sigma$, one starting with $a^{-1}$ and the other starting with $b^{-1}$.

From the construction of $E_{i}, E_{i} W_{\frac{p}{q}}^{-1}$ (as reduced word) always starts with $b^{-1}$ and $E_{i} W_{\frac{p}{q}}$ always starts with a letter different from $b^{-1}$. Therefore, $z_{i}$ has two 
different representations in $\Sigma$, one starting with $b^{-1}$ (corresponding to $\overline{E_{i} W_{\frac{p}{q}}^{-1}}$ ) and the other starting with a different letter. Therefore,

$$
z_{i}=\psi_{\frac{p}{q}}\left(x_{i}\right)=\psi_{\frac{p}{q}}\left(y_{i}\right)
$$

where $x_{i}$ and $y_{i}$ are endpoints of a leaf $l_{i}$ of $\lambda_{\frac{p}{q}}$, intersecting the side $\gamma_{b^{-1}}$ of the fundamental domain.

With the previous arguments, we obtain the following theorem:

Theorem 3.7. The core chain of circles separates the complex plane into the four regions consisting of words starting with $a, b, a^{-1}$, and $b^{-1}$. In particular, the tangency points $z_{i}$ between $C_{i}$ and $C_{i+1}$ are of the form:

$$
z_{i}=\varpi \frac{p}{q}\left(\overline{X_{i}}\right)=\varpi_{\frac{p}{q}}\left(\overline{Y_{i}}\right),
$$

where $\overline{X_{i}}, \overline{Y_{i}} \in \Sigma, e_{1}\left(X_{i}\right)=b^{-1}$ and $e_{1}\left(Y_{i}\right) \neq b^{-1}$.

Figure 1 and Figure 2 visualize this theorem.

\section{The MANifolds $N_{n}$ AND THEIR Limit $N$}

In this section we want to introduce a sequence of double-cusp groups $G_{n}$.

Let $\xi \in \mathbb{R} \backslash \mathbb{Q}$ be an irrational number. We want to define a sequence of rational numbers $\frac{p_{n}}{q_{n}}$ tending to $\xi$. If $\xi$ is written as an infinite continued fraction,

$$
\xi=\frac{1}{\alpha_{1}+\frac{1}{\alpha_{2}+\frac{1}{\alpha_{3}+\frac{1}{\alpha_{4}+\frac{1}{\ldots}}}}}:=\left[\alpha_{1}, \alpha_{2}, \alpha_{3}, \alpha_{4}, \ldots\right],
$$

then $\frac{p_{n}}{q_{n}}$ is the rational number given by the finite continued fraction,

$$
\frac{p_{n}}{q_{n}}=\left[\alpha_{1}, \alpha_{2}, \alpha_{3}, \alpha_{4}, \ldots, \alpha_{n}\right] .
$$

Let $G_{n}$ be the double-cusp group with end invariants $\left(\frac{1}{0}, \frac{p_{n}}{q_{n}}\right)$. Following the previous notation, $G_{n}=G_{\frac{p_{n}}{q_{n}}}$, but for simplicity now we just denote $G_{n}$, considering $\xi$ fixed.

In this group, the elements corresponding to the rationals $\frac{1}{0}$ and $\frac{p_{n}}{q_{n}}$ are accidental parabolics.

It is proved in 22 that the pivot sequence of the group $G_{n}$ is determined by the continued fraction expansion for the number $\frac{p_{n}}{q_{n}}$, therefore it is possible to define the rationals $\frac{p_{n}}{q_{n}}$ by the Farey triangulation.

Let $N_{n}=\mathbb{H}^{3} / G_{n}$ be hyperbolic 3-manifold.

We denote by $\Lambda_{n}$ the limit set and by $\Omega_{n}=\hat{\mathbb{C}} \backslash \Lambda_{n}$ the ordinary set. Since both end invariants $\frac{1}{0}$ and $\frac{p_{n}}{q_{n}}$ are rational, then the group is geometrically finite. Each component of $\Omega_{n} / G_{n}$ is a thrice-punctured sphere $S_{n}$. Topologically one can think of the geometrically finite end related to $\frac{p_{n}}{q_{n}}$ as

$$
S_{n} \times[-1,1]-\left\{\text { a simple closed curve corresponding to } \frac{p_{n}}{q_{n}}\right\} \times\{1\} .
$$

Now we can consider $G_{n}$ as a sequence of groups, that is, we fix $\xi$ and vary the number $n$ of elements in the continued fraction. Then from Theorem 2.12 we have the following proposition. 
Proposition 4.1. Let $G_{n}$ be as above. Then

$$
G_{n} \stackrel{a l g}{\longrightarrow} G_{\infty}
$$

where $G_{\infty}=\left(\frac{1}{0}, \xi\right)$.

Let $N_{\infty}=\mathbb{H}^{3} / G_{\infty}$ be the limiting hyperbolic 3-manifold. Since the end invariants of $G_{\infty}$ are $\left(\frac{1}{0}, \xi\right)$, then the group $G_{\infty}$ is geometrically infinite (because $\xi \in \mathbb{R} \backslash \mathbb{Q}$ ). Let $\Lambda$ be the limit set of $G_{\infty}$ and $\Omega=\mathbb{H}^{2} \backslash \Lambda$.

$N_{\infty}$ is singly degenerate because $\Omega / G_{\infty}$ has just one component that is a thricepunctured sphere $S$. Topologically $\left(\mathbb{H}^{3} \cup \Omega\right) / G_{\infty}$ is the product $S \times[0,1)$.

The next theorem is a consequence of the ending lamination theorem for oncepunctured tori (proved in [22]) and of the Canary-Thurston covering theorem.

Theorem 4.2. Let $N_{n}$ and $N_{\infty}$ be as above. Then

$$
N_{n} \stackrel{g e o}{\longrightarrow} N_{\infty} \text {. }
$$

\section{Behaviour of the Circles}

5.1. The first circle of the chain. Let $C_{n, j}$ be the circle chain for the group $G_{n}$ with end invariants $\left(\frac{1}{0}, \frac{p_{n}}{q_{n}}\right)$. We want to prove that the radius of the first circle of the chain decreases when $n$ tends to infinity.

Theorem 5.1. The radius $r_{n, 0}$ of $C_{n, 0}$ satisfies $r_{n, 0} \sim \frac{1}{n}$. In particular, $r_{n, 0}$ tends to 0 when $n \rightarrow \infty$.

Proof. Let us consider the manifold $N_{n}$ given by $\mathbb{H}^{3} / G_{n}$. Let $X_{n}$ be the convex core of $N_{n}$. Let $\Lambda_{n}$ be the limit set and let $\Omega_{n}$ be the ordinary set.

Since $N_{n}$ is a double-cusp group, $\Omega_{n} / G_{n}$ is divided into 2 thrice-punctured spheres $\Omega_{n}^{+} / G_{n}$ and $\Omega_{n}^{-} / G_{n}$, and each $\Omega_{n}^{+}$and $\Omega_{n}^{-}$is an infinite union of disks in the complex plane. Since each component of the boundary of the convex hull $\partial C H_{n}(\Lambda)$ corresponds to a component of $\Omega_{n}$, the boundary of the convex core $X_{n}$ has two components $\partial X_{n}^{-}$and $\partial X_{n}^{+}$that are 2 thrice-punctured spheres. Notice that the main cusp in $\partial X_{n}^{-}$is exactly the same as the one in $\partial X_{n}^{+}$(since the commutator $K$ of the 2 generators $a$ and $b$ is also the commutator of the elements $W_{\frac{p_{n}}{q_{n}}}$ and $\left.W \frac{p_{n+1}}{q_{n+1}}\right)$.

Between the infinite union of tangent circles corresponding to $\Omega_{n}^{-}$in the complex plane, let us consider the disk $R$ that is tangent to $C_{n, 0}$ in -1 . The Fuchsian group generated by $a$ and $K$ stabilizes $R$. With the normalisation described previously for defining the group $G_{n}, R$ is the half plane in $\mathbb{C}$ given by $\left\{z=z_{1}+i z_{2} \in \mathbb{C}: z_{2} \leq 0\right\}$ (in fact $K(\hat{\mathbb{R}})=\hat{\mathbb{R}}$ and $a(\hat{\mathbb{R}})=\hat{\mathbb{R}})$. Notice that $R$ does not depend on $n$.

Using [22, the manifold $N_{n}$ is quasi-isometric to the model manifold $M_{n}$. The construction of $M_{n}$ only depends on the end invariants of $N_{n}$. More precisely, given the end invariants we can easily construct the so-called pivot sequence (from the Farey triangulation) and then build $M_{n}$ by piling up blocks one-by-one, each of these with a metric depending on the pivot sequence. In particular, there exists a quasi-isometry

$$
\tilde{f}: \tilde{M}_{n} \longrightarrow \tilde{N}_{n}
$$

between their universal covers and therefore there exist universal constants $k, \delta$ such that $\forall x_{1}, x_{2} \in M_{n}$ we have

$$
\frac{1}{k} d\left(\widetilde{x_{1}}, \widetilde{x_{2}}\right)+\delta \leq d\left(\tilde{f}\left(\widetilde{x_{1}}\right), \tilde{f}\left(\widetilde{x_{2}}\right)\right) \leq k d\left(\widetilde{x_{1}}, \widetilde{x_{2}}\right)+\delta .
$$


For more details about the construction of the model manifold $M_{n}$, one can see [22].

We shall work with $M_{n}$ and then transfer the result in $N_{n}$ by $f$.

Let us consider a point $x \in \partial X_{n}^{-}$in $M_{n}$ such that $x \in B_{\sigma^{e}}\left(\frac{1}{4}, p\right) \backslash B_{\sigma^{e}}\left(\frac{1}{8}, p\right)$ in $\partial X_{n}^{-}$where $p$ is the main cusp and $B_{\sigma^{e}}(b, p)$ is the $\sigma^{e}$-neighbourhood of radius $b$ of $p$ (the metric $\sigma^{e}$ is the metric in the model defined in [22]).

Then $f(x)$ has a lift $\tilde{x}$ in the hemisphere $H_{R}$ over $R$ in $\mathbb{H}^{3}$ such that $\tilde{x}$ is far from the main cusp (this means that $\tilde{x}$ is far from -1 ). Using the coordinates $a=a_{1}+a_{2} i+a_{3} j$ for a point $a$ in $\mathbb{H}^{3}$, we can suppose that $\tilde{x}=-1+\frac{1}{6} j$.

Let $\gamma$ be the vertical path in $M_{n}$ starting from $x$ and let $y$ be the endpoint of $\gamma$ in $\partial X_{n}^{+}$. Then $y \in B_{\sigma^{e}}\left(\frac{1}{4}, p\right) \backslash B_{\sigma^{e}}\left(\frac{1}{8}, p\right)$ and $\gamma$ is in the boundary of the Margulis tube corresponding to the parabolic commutator $K$. Since $f$ sends Margulis tubes to Margulis tubes by construction, then $f(\gamma) \in N_{n}$ is in the boundary of the Margulis tube $T_{K}$ in $N_{n}$. From the definition, $\tilde{f}$ is a quasi-isometry and it is far to be an isometry, especially near the boundary of the main cusp of the model. Hence, by the construction of the model manifold and since the path $\gamma$ is in the boundary of the Margulis tube corresponding to $K$, then

$$
l_{M_{n}}(\gamma) \sim l_{N_{n}}(f(\gamma)) .
$$

Let $\tilde{\gamma}$ be the lift of $f(\gamma)$ starting from $\tilde{x}$ and let $\tilde{y}$ be the endpoint of $\tilde{\gamma}$ in the hemisphere $H_{C_{n, 0}}$ over $C_{n, 0}$ in $\mathbb{H}^{3}$ (we have that $\tilde{y} \in H_{C_{n, 0}}$ for the choice of $\gamma$ ). Since $x$ and $y$ are in the same neighbourhood of the cusp $p$, then $\tilde{y}$ belongs to the horosphere $H\left(\frac{1}{6},-1\right)$ of Euclidean radius $\frac{1}{6}$ and tangent to $\mathbb{C}$ at -1 .

Since $\gamma$ is a vertical path joining points in the two boundaries of the convex core, then $l_{M_{n}}(\gamma)=n+1$ (in the product metric of $M_{n}$, the vertical distance between the two boundaries of the convex core is $n+1$ since the model manifold is made by $n+1$ blocks each of which has height 1 ). Therefore the hyperbolic length of the path $f(\gamma)$ is

$$
l_{N_{n}}(f(\gamma)) \sim n+1
$$

Using $l_{N_{n}}(f(\gamma))$, we want to find the hyperbolic distance between $\tilde{x}$ and $\tilde{y}$, i.e. the length of the geodesic $\tilde{\varrho}$ between $\tilde{x}$ and $\tilde{y}$.

Lemma 5.2. The hyperbolic distance between $i$ and $i+n$ is

$$
d=d_{\mathbb{H}^{2}}(i, i+n) \sim 2 \ln n,
$$

when $n$ tends to infinity.

Proof. Using the well-known formula

$$
\cosh d\left(z_{1}, z_{2}\right)=1+\frac{\left|z_{1}-z_{2}\right|^{2}}{2 \Im z_{1} \Im z_{2}}
$$

we obtain that

$$
\cosh d=1+\frac{n^{2}}{2}
$$

and therefore

$$
\frac{e^{d}+e^{-d}}{2}=1+\frac{n^{2}}{2} .
$$

When $n$ tends to infinity, then $d \sim 2 \ln n$. 
Using the previous lemma, we obtain that

$$
l_{\mathbb{H}^{3}}(\tilde{\varrho}) \sim 2 \ln \left(l_{N_{n}}\right) \sim 2 \ln (n+1)=\ln (n+1)^{2} .
$$

Therefore the hyperbolic distance between $\tilde{x}$ and $\tilde{y}$ is of the order of $\ln (n+1)^{2}$.

Lemma 5.3. Let $\tilde{x}$ and $\tilde{y}$ be as above. Then the radius $r_{n, 0} \sim\left(\frac{1}{n}\right)$.

Proof. Let us consider the plane $P$ in $\mathbb{H}^{3}$ perpendicular to the boundary $\hat{\mathbb{C}}$ and containing the geodesic $\tilde{\varrho}$.

Let $I_{R}$ be the intersection between $P$ and $H_{R}$ and let $I_{C_{n, 0}}$ be the intersection between $P$ and $H_{C_{n, 0}}$. Then $I_{R}$ is a geodesic half-line perpendicular to the boundary of $P$ in 0 and $I_{C_{n, 0}}$ is a geodesic semi-circle perpendicular to $P$ in 0 (see Figure 5 ).

To simplify the calculation we can apply a translation that sends -1 to 0 .

Considering $P$ as the hyperbolic plane, in these new coordinates, we have that $\tilde{x}=\frac{1}{6} i$ and $\tilde{y}=y_{1}+i y_{2}$.

Let $c$ be the centre of $I_{C_{n, 0}}$ of radius $r$.

The order of $r$ is the same as the order of $r_{n, 0}$ since $\tilde{y}$ is in a neighbourhood of the highest point $\tilde{z}$ of $H_{C_{n, 0}}$. In fact we chose $\tilde{\varrho}$ to be the shortest path between $\tilde{x}$ and $\tilde{y}$ and therefore $\tilde{\varrho}$ has to end in the closest lift of $y$ to $\tilde{x}$, that is, in a neighbourhood of $\tilde{z}$. Therefore, we want to estimate $r$.

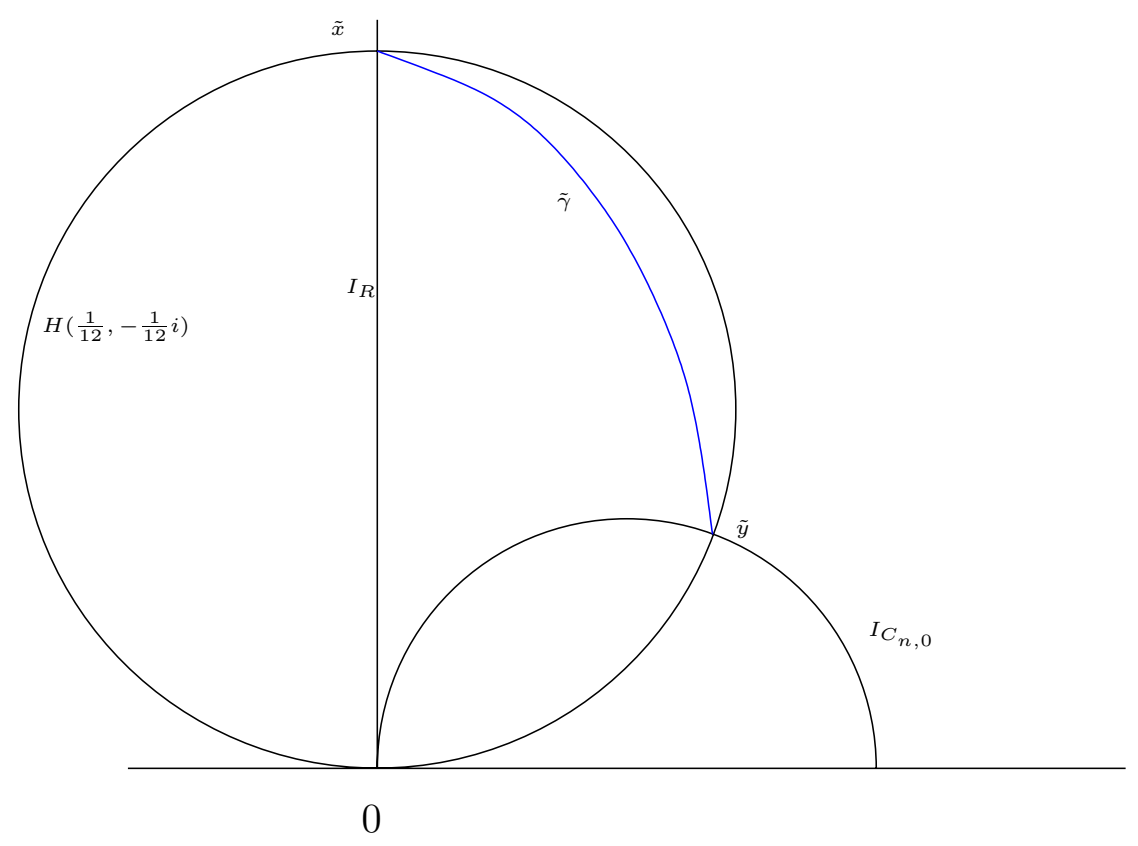

Figure 5. The plane $P$ 
Since $0 \in I_{C_{n, 0}}$, then

$$
r=c .
$$

For $\tilde{y} \in I_{C_{n, 0}}$, then

$$
\left(y_{1}-c\right)^{2}+y_{2}^{2}=c^{2} .
$$

Let $H\left(\frac{1}{12}, \frac{1}{12} i\right)$ be the horodisk in $P$ of centre $\frac{1}{12} i$ and radius $\frac{1}{12}$.

Since $y$ is in the boundary of the $\frac{1}{6}$-neighbourhood of $p$, then $\tilde{y} \in H\left(\frac{1}{12}, \frac{1}{12} i\right)$ and then

$$
\left(y_{1}\right)^{2}+\left(y_{2}-\frac{1}{12}\right)^{2}=\frac{1}{144}
$$

Since $l_{\mathbb{H}^{3}}(\tilde{\varrho})=\ln (n+1)^{2}$, then

$$
\cosh \left(\ln (n+1)^{2}\right)=1+\frac{|\tilde{x}-\tilde{y}|^{2}}{2 \Im \tilde{x} \Im \tilde{y}}=1+\frac{\left(y_{1}\right)^{2}+\left(\frac{1}{6}-y_{2}\right)^{2}}{\frac{y_{2}}{3}} .
$$

Combining Equation (4) and Equation (5), we obtain

$$
\frac{y_{2}}{3}\left(\cosh \left(\ln (n+1)^{2}\right)-1\right)=-y_{2}^{2}+\frac{1}{6} y_{2}+y_{2}^{2}-\frac{1}{3} y_{2}+\frac{1}{36} .
$$

This equation in $y_{2}$ of the first degree has the following solution:

$$
y_{2}=\frac{1}{12 \cosh \left(\ln (n+1)^{2}\right)-6} .
$$

Therefore

$$
y_{2} \sim\left(\frac{1}{n^{2}}\right) .
$$

Using Equations (3), (2) and (4) we obtain that

$$
r_{n, 0} \sim\left(\frac{1}{n}\right) .
$$

From the lemma, $r_{n, 0} \sim\left(\frac{1}{n}\right)$ then obviously the radius of $C_{n, 0}$ tends to 0 when $n \rightarrow \infty$ and therefore the theorem is proved.

5.2. A bit of symmetry. In this section we prove that not only the radius of the first circle tends to zero, but also the radii of the other circles decrease.

Proposition 5.4. Let $r_{p}$ be the radius of $C_{n, p}$ and $r_{q}$ be the radius of $C_{n, q}$. Then $r_{p}=r_{q}=r_{0}$.

Proof. Let $\hat{\varphi}$ be the Möbius transformation given by $\hat{\varphi}=a b-b a=\left(\begin{array}{cc}-2 i & 2 i \mu \\ 0 & 2 i\end{array}\right)$.

It is easy to see that the trace of $\hat{\varphi}$ is 0 and that $\hat{\varphi}$ satisfies the following relations: $\hat{\varphi} a \hat{\varphi}=a^{-1}$ and $\hat{\varphi} b \hat{\varphi}=b^{-1}$. This transformation was introduced by Jørgensen in [11.

We can normalize the determinant of $\hat{\varphi}$ to be 1 , to obtain the transformation $\varphi=\left(\begin{array}{cc}-i & i \mu \\ 0 & i\end{array}\right)$, so that $\varphi$ acts on the complex plane $\mathbb{C}$ as $\varphi(z)=-z+\mu$. Then $\varphi$ is the hyperelliptic involution with $\varphi^{2}=I, \varphi a \varphi=a^{-1}, \varphi b \varphi=b^{-1}$ and the trace of $\varphi$ is 0 . Solving the equation $\varphi(z)=z$ we can notice that $\varphi$ has two fixed points: $\frac{\mu}{2}$ and $\infty$. 
Moreover, $\varphi$ and $a(z)=z+2$ are Euclidean isometries since $a$ is a translation and $\varphi$ is a rotation of $\pi$ around the fixed point $\frac{\mu}{2}$.

By the definition of the circle chain $C_{j}, C_{q}=a\left(C_{0}\right)$ and $C_{p}=a^{-1}\left(C_{p+q}\right)=$ $a^{-1} \varphi\left(C_{0}\right)$. Therefore $r_{0}=r_{p}=r_{q}$ (because distance is preserved by $a$ and $\varphi$ ).

\section{Corollary 5.5.}

(1) $r_{p} \sim \frac{1}{n}$, in particular, $r_{p}$ tends to 0 when $n \rightarrow \infty$.

(2) $r_{q} \sim \frac{1}{n}$, in particular, $r_{q}$ tends to 0 when $n \rightarrow \infty$.

Proof. From lemma 5.3 we know that $r_{0} \sim\left(\frac{1}{n}\right)$ and from the previous proposition we know that $r_{p}=r_{q}=r_{0}$, therefore $r_{p}=r_{q} \sim\left(\frac{1}{n}\right)$, and then $r_{p}=r_{q}$ tends to 0 when $n \rightarrow \infty$.

Let us denote by $r_{j}$ the radius of the circle $C_{n, j}$.

From the following proposition we have to study only the circles $C_{i}$ for $0 \leq i \leq \frac{p}{2}$ and $p \leq i \leq\left[\frac{q-p}{2}\right]$.

\section{Proposition 5.6.}

(1) For $0 \leq l<\frac{p}{2}$ we have $r_{p-l}=r_{l}$.

(2) For $0 \leq l<\left[\frac{q-p}{2}\right]$ we have $r_{q-l}=r_{p+l}$.

Proof. As in the previous proposition, we define $\varphi=\left(\begin{array}{cc}-i & i \mu \\ 0 & i\end{array}\right)$, that is, $\varphi(z)=$ $-z+\mu$. Since $\varphi$ is a rotation of angle $\pi$ around the point $\frac{\mu}{2}$ (the tangency point between the circles $C_{\left[\frac{q-p}{2}\right]+p}$ and $C_{\left[\frac{q-p}{2}\right]+p+1}$ ), and since $\varphi$ satisfies $\varphi^{2}=I, \varphi a \varphi=$ $a^{-1}, \varphi b \varphi=b^{-1}$, then $\varphi$ creates a rotation symmetry, as stated, between the circles.

Corollary 5.7. The radius $r_{n, j}$ of the circle $C_{n, j}$ tends to 0 when $n \rightarrow \infty$ for all $j$.

Proof. Let $\tilde{x}$ and $\tilde{y}$ be points in the two boundaries of the convex core as in Theorem 5.1 and let $\gamma_{n, j}$ be the curve joining $\tilde{x}$ and $\tilde{y}$ in the homotopy class of $E_{n, j}$ where

$$
C_{n, j}=E_{n, j}\left(C_{n, 0}\right) .
$$

Let $\gamma$ be the "vertical" path (defined in Theorem 5.1) joining $\tilde{x}$ with $\tilde{y}$.

Among all the curves (in any homotopy class) joining $\tilde{x}$ to $\tilde{y}$, the path $\gamma$ is the shortest since it is the one crossing each block in the shortest way (the vertical one) and each path joining the two boundaries of the convex core has to cross each block.

Therefore, we obtain that

$$
l(\gamma) \leq l\left(\gamma_{n, j}\right) .
$$

Therefore, $r_{n, j} \leq r_{n, 0}$ and since $r_{n, 0} \longrightarrow 0$ when $n \rightarrow \infty$, then

$$
r_{n, j} \longrightarrow 0 \text {. }
$$

\section{Application of McShane's identity}

In this section, we use the results of Theorem 5.1 to find an application of McShane's identity. This identity was introduced by McShane in [19] and it involves the lengths of simple closed curves on a punctured torus. It was extended by Bowditch in [7] to hyperbolic 3-manifolds and it was used by Akiyoshi, Miyachi, 
and Sakuma in [1] for the width of the limit set. We will refer to some definitions and results of [1].

Using Jørgensen's normalisation of the once-punctured torus $(K(z)=z-2)$, the limit set of the group is contained in a band of a certain height (twice the width). We prove that this height is proportional to the number $n$ of the pivots. Figure 6 shows evidence of this result. In the figure, we superimpose five different chains of circles (each of them has a different colour), each time increasing by one the number of pivots.

6.1. The width of the limit set. We begin with some definitions and a theorem that can be found in [1]. The proposition is the first consequence of our work in this setting.

Let $S C C$ be the set of homotopy classes of (non-peripheral) simple closed curves on a punctured torus $T(S)$. We have already seen in Section 2 that $S C C$ is identified with $\hat{\mathbb{Q}}$. Let $\lambda(\gamma)$ be the complex translation length of an element $\gamma \in S C C$.

McShane's identity, proved by Bowditch for quasi-Fuchsian punctured torus groups in [7], is the following:

$$
\sum_{\gamma \in S C C} \frac{1}{1+e^{\lambda(\gamma)}}=\frac{1}{2}
$$

Let $\left(\frac{1}{0}, \frac{p_{n}}{q_{n}}\right)$ be the end invariants of the manifold $N_{n}=\mathbb{H}^{3} / G_{n}$. Then $S^{1}-$ $\left\{\frac{1}{0}, \frac{p_{n}}{q_{n}}\right\}$ has two components $F_{L}$ and $F_{R}$. Let $S_{L}=F_{L} \cap \hat{\mathbb{Q}}$ and $S_{R}=F_{R} \cap \hat{\mathbb{Q}}$ be the intersections of the two components $F_{L}$ and $F_{R}$ with $\mathbb{Q}$.

It follows immediately from Equation (6) (see [1]) that:

$$
\Im \sum_{\gamma \in S_{L}} \frac{1}{1+e^{\lambda(\gamma)}}=-\Im \sum_{\gamma \in S_{R}} \frac{1}{1+e^{\lambda(\gamma)}} .
$$

Now we want to relate this equality with the width of the limit set. For every parabolic element $p \in G_{n}$, we can define the width $\omega_{p}(\Lambda)$ of the limit set related to $p$.

Let $C\left(N_{n}\right)$ be the convex core of the manifold $N_{n}$. Choosing a cusp neighbourhood $T_{p}$ of $p$, let $A_{p}=\partial T_{p} \cap C\left(N_{n}\right)$ be an Euclidean annulus (the Euclidean metric is defined up to scaling). Then the width $\omega_{p}(\Lambda)$ related to $p$ is equal to the modulus of $A_{p}$, that is:

$$
\omega_{p}(\Lambda)=\frac{\left(\text { Euclidean distance between the components of } \partial A_{p}\right)}{\left(\text { Euclidean length of a component of } \partial A_{p}\right)} .
$$

Theorem 6.1 (Theorem 5.1 of [1]). Let $K$ be the parabolic commutator of the two generators $a$ and $b$.

Then for any geometrically finite punctured torus group, the width $\omega_{K}(\Lambda)$ of the limit set $\Lambda$ related to $K$ is given by the following identity:

$$
\omega_{K}(\Lambda)=\Im \sum_{\gamma \in S_{L}} \frac{1}{1+e^{\lambda(\gamma)}}=-\Im \sum_{\gamma \in S_{R}} \frac{1}{1+e^{\lambda(\gamma)}} .
$$

It is possible to give an equivalent definition of $\omega_{p}(\Lambda)$ in terms of the choice of coordinates. Let us choose a normalisation such that the parabolic transformation 


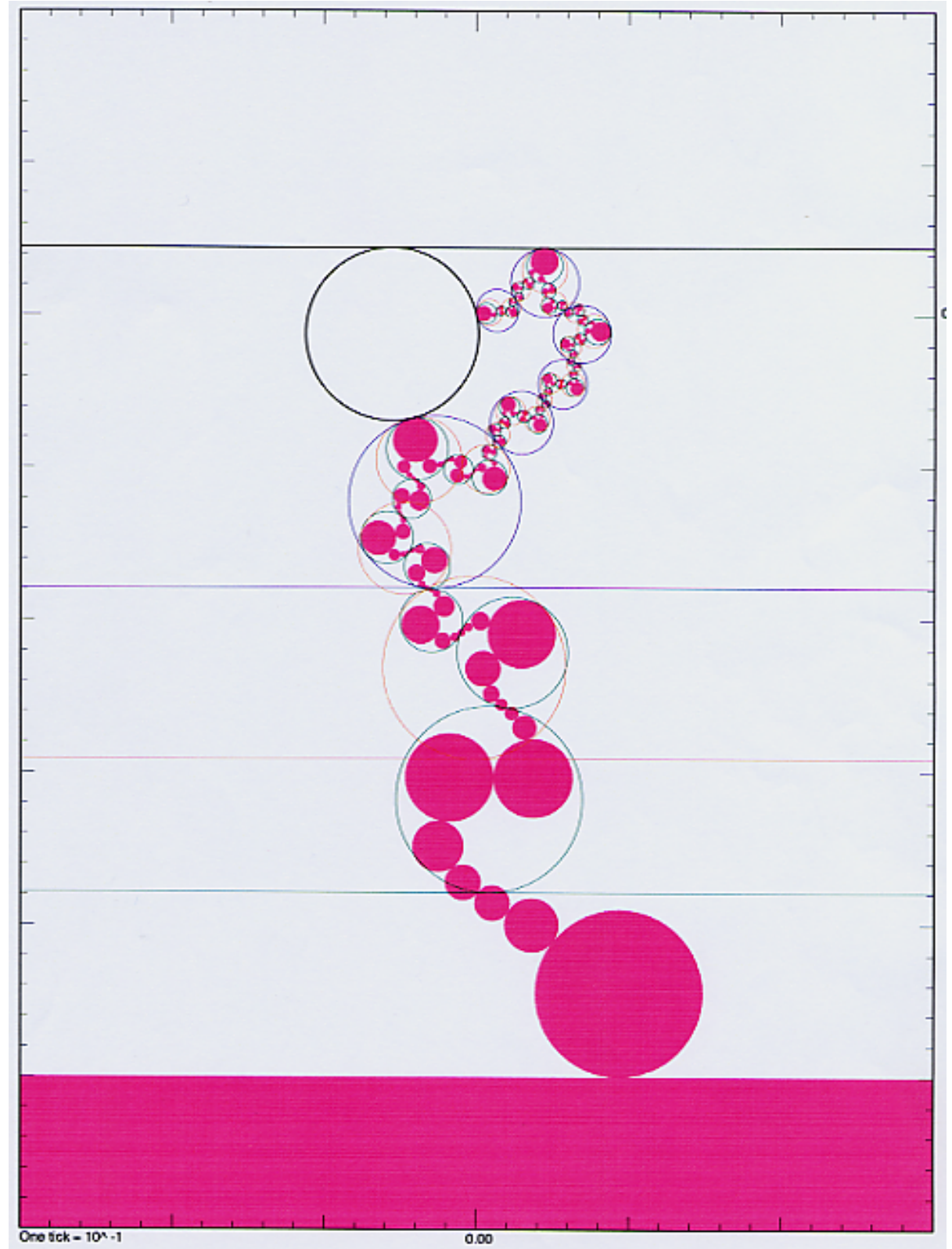

FiguRE 6. Jørgensen's normalisation

$p$ is a translation by 2 and therefore the fixed point of $p$ is $\infty$. Then the width $\omega_{p}(\Lambda)$ of the limit set satisfies the following equation:

$$
\omega_{p}(\Lambda)=\frac{1}{2}\left(m_{p}^{+}(\Lambda)-m_{p}^{-}(\Lambda)\right)
$$

where

$$
m_{p}^{+}(\Lambda)=\max \{\Im z \text { such that } z \in \Lambda \cap \mathbb{C}\}
$$


and

$$
m_{p}^{-}(\Lambda)=\min \{\Im z \text { such that } z \in \Lambda \cap \mathbb{C}\} .
$$

For the proof of the equality (7), one can see [1].

Proposition 6.2. Let us choose the normalisation such that $a(z)=z+2$ and $b_{\mu}(z)=\frac{\mu z+1}{z}$. Then

$$
\omega_{a}(\Lambda)=\frac{\Im(\mu)}{2} .
$$

Proof. We fixed a normalisation such that the two generators had the expression $a=\left(\begin{array}{ll}1 & 2 \\ 0 & 1\end{array}\right)$ and $b_{\mu}=\left(\begin{array}{cc}-i \mu & -i \\ -i & 0\end{array}\right)$. Therefore, the parabolic commutator $K$ is $K=b^{-1} a^{-1} b a=\left(\begin{array}{cc}1 & 2 \\ -2 & -3\end{array}\right)$ and its fixed point is -1 . With this choice of coordinates, $a(z)=z+2$ and $a(\infty)=\infty$.

Using the definition of the width of the limit set in terms of the normalisation, we can easily find $\omega_{a}(\Lambda)$.

The distance between the two lines containing the limit set is $\Im(\mu)$ (in fact, in this normalisation, $C_{p}$ is tangent to the line $\Im(z)=\Im(\mu)$ ), i.e. $m_{a}^{+}(\Lambda)-m_{a}^{-}(\Lambda)=\Im(\mu)$, then $\omega_{a}(\Lambda)=\frac{\Im(\mu)}{2}$.

6.2. McShane's identity in terms of the pivots. In this section, we want to find a relation between McShane's identity and the radius $r_{0}$ of the circle $C_{0}$ and our estimates in terms of the number of the pivots. For this aim, we calculate the width of the limit set related to the commutator $K=b^{-1} a^{-1} b a$.

Theorem 6.3. Let $K$ be the commutator of the two generators $a$ and $b$ and let $r_{0}$ be the radius of $C_{0}$.

Then

$$
\omega_{K}(\Lambda)=\frac{1}{4 r_{0}} .
$$

Proof. To calculate $\omega_{K}(\Lambda)$, we have to find a normalisation $\tilde{a}, \tilde{b}$ such that the new commutator $\tilde{K}=\tilde{b}^{-1} \tilde{a}^{-1} \tilde{b} \tilde{a}: z \mapsto z-2$.

Since

$$
K(z)=\frac{z+2}{-2 z-3},
$$

then $K$ has -1 as a fixed point. Therefore, we have to find a Möbius transformation that sends -1 to $\infty$.

Let $v$ be the transformation sending -1 to $\infty$ and satisfying

$$
v(z)=\frac{z}{z+1} \text {. }
$$

Then $v=\left(\begin{array}{ll}1 & 0 \\ 1 & 1\end{array}\right)$, and $v^{-1}=\left(\begin{array}{cc}1 & 0 \\ -1 & 1\end{array}\right)$.

Using the matrix $v$, we define the new generators $\tilde{a}$ and $\tilde{b_{\mu}}$.

The transformation $\tilde{a}=v a v^{-1}$ satisfies

$$
\tilde{a}(z)=\frac{-z+2}{-2 z+3} .
$$

The transformation $\tilde{b}_{\mu}=v b v^{-1}$ satisfies

$$
\tilde{b_{\mu}}(z)=\frac{(-1+\mu) z+1}{\mu z+1} .
$$


Finally, the commutator $\tilde{K}=v K v^{-1}$ satisfies

$$
\tilde{K}(z)=z-2 .
$$

Since $v$ has real entries and since the image of a line by a Möbius transformation is a line or a circle, then $v(\mathbb{R})=\mathbb{R}$.

The circle $C_{0}$ and the line $\mathbb{R}$ are tangent at the point -1 and $v(-1)=\infty$, then $v\left(C_{0}\right)$ and $v(\mathbb{R})$ are tangent at $\infty$, and hence $v\left(C_{0}\right)$ is a horizontal line.

Then the limit set is contained in the band between $v\left(C_{0}\right)$ and $v(\mathbb{R})$. Therefore, to find the width of the limit set we just have to find the equation of the horizontal line $v\left(C_{0}\right)$.

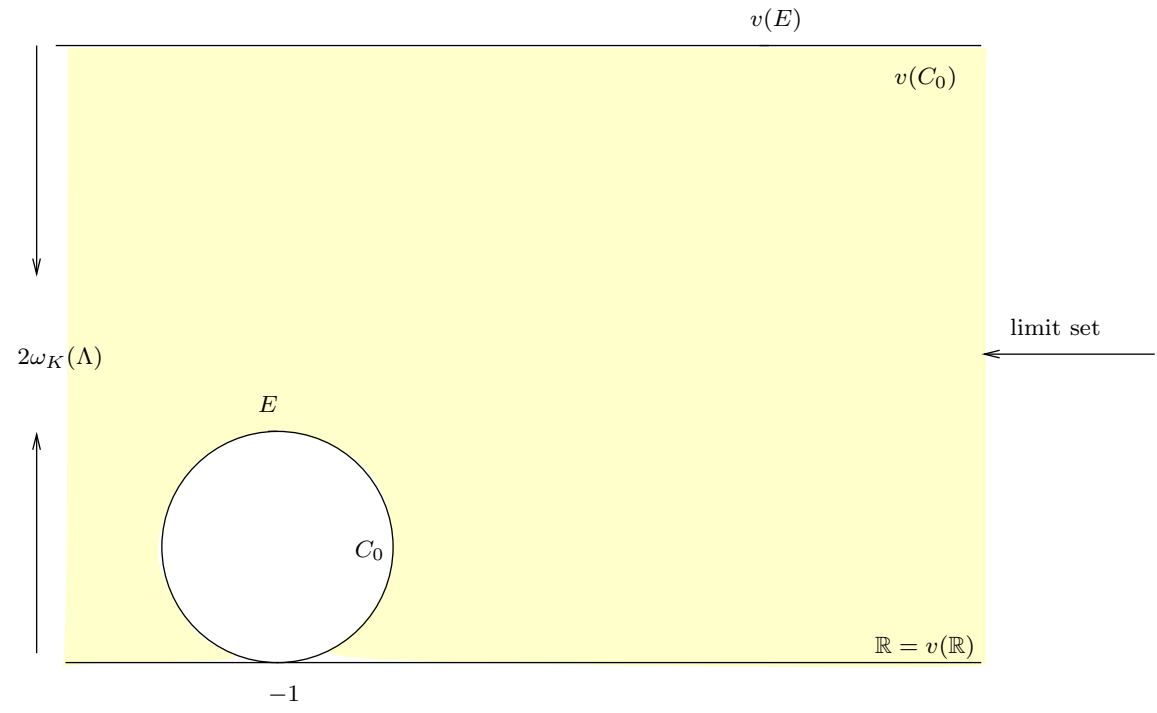

Figure 7 . The width of the limit set

For example, let $E$ be the point in $C_{0}$ of coordinates $E=-1+2 i r_{0}$, then $v(E) \in v\left(C_{0}\right)$ and $v(E)=v\left(-1+2 i r_{0}\right)=1+\frac{i}{2 r_{0}}$. Therefore, $v\left(C_{0}\right)$ is defined by the equation in the complex plane $y=\frac{1}{2 r_{0}}$ and

$$
d\left(v\left(C_{0}\right), v(\mathbb{R})\right)=\frac{1}{2 r_{0}} .
$$

Since

$$
\omega_{K}(\Lambda)=\frac{1}{2}\left(m_{K}^{+}(\Lambda)-m_{K}^{-}(\Lambda)\right)=\frac{1}{2}\left(d\left(v\left(C_{0}\right), v(\mathbb{R})\right)\right),
$$

then

$$
\omega_{K}(\Lambda)=\frac{1}{4 r_{0}}
$$

Corollary 6.4. Let $n$ be the number of pivots for the end invariants $\left(\frac{1}{0}, \frac{p_{n}}{q_{n}}\right)$ of the manifold $N_{n}=\mathbb{H}^{3} / G_{n}$, then

$$
\Im \sum_{\gamma \in S_{L}} \frac{1}{1+e^{\lambda(\gamma)}}=-\Im \sum_{\gamma \in S_{R}} \frac{1}{1+e^{\lambda(\gamma)}} \sim n .
$$


Proof. This corollary is a straightforward consequence of the previous theorem and of Theorem 5.1.

Since

$$
\omega_{K}(\Lambda)=\Im \sum_{\gamma \in S_{L}} \frac{1}{1+e^{\lambda(\gamma)}}=-\Im \sum_{\gamma \in S_{R}} \frac{1}{1+e^{\lambda(\gamma)}}
$$

and since

$$
\omega_{K}(\Lambda)=\frac{1}{4 r_{0}},
$$

then we can use the estimate (proved in Theorem 5.1)

$$
r_{0} \sim \frac{1}{n}
$$

to obtain that

$$
\omega_{K}(\Lambda) \sim n
$$

\section{THE CASE $n=3$}

In this section we focus our attention on the case in which $p=1$. Therefore, we study the double-cusp group $G=\left(\frac{1}{0}, \frac{1}{q}\right)$, with more interest in the situation where $q$ is large.

The choice of this example is due to the fact that if $p=1$, then the combinatorics of the circle chain is very simple and the pivot sequence is short. In this case $n=3$, therefore, the estimates in Section 5 do not make sense for $G$. Nevertheless, we find other results for the radii of the circles $C_{j}$.

7.1. The model manifold and the estimates for the Margulis tubes. When $p=1$, the Pivot sequence is made by 3 elements $(n=3)\left\{\frac{1}{0}, \frac{0}{1}, \frac{1}{q}\right\}$ corresponding to the three words $W_{\frac{1}{0}}=a, W_{\frac{0}{1}}=b$, and $W_{\frac{1}{q}}=a^{-1} b^{q}$.

In Figure 8 we depict part of the Farey triangulation of the Poincaré disk for the case $q=6$. Notice that there are $q-2$ edges starting from the pivot $\frac{0}{1}$ and separating the two end invariants $\frac{1}{0}$ and $\frac{1}{q}$. Therefore, the width $\omega(b)$ of the pivot $b$ is $q-1=5$ (in fact $\frac{1}{6}=D_{\frac{0}{1}}^{5}\left(\frac{1}{0}\right)$ ).

The model manifold $M=\bigcup_{n=0}^{2} B_{n}$ has three blocks $B_{n}$ (see Figure 9), two boundary blocks $B_{0}$ and $B_{2}$ and one internal block $B_{1}$, where $B_{0}=B_{a, b}, B_{1}=$ $B_{b, a, a^{-1} b^{q}}$ and $B_{2}=B_{a^{-1} b^{q}, b}$.

In each block we can put the metric $\sigma^{e}$ described in 22. This means that the first block $B_{0}$ contains the boundary Margulis tube $T_{a}$ of the pivot $a$ and outside $T_{a}$ the length of $a$ and $b$ has order 1 . The same happens for $B_{2}$, interchanging $a$ with $a^{-1} b^{q}$. In the internal block $B_{1}$, we have the Margulis tube $T_{b}$ of $b$ and outside it the length of the three pivots has order 1 .

With the Pivot Theorem [22, Minsky gives an estimate of the complex length of a pivot $\alpha_{n}$ knowing the width of $\alpha_{n}: \frac{2 \pi i}{\lambda\left(\alpha_{n}\right)} \doteq \omega\left(\alpha_{n}\right)+i$ (the symbol $\doteq$ means a bound on hyperbolic distance in $\mathbb{H}^{2}$ between the left and right sides, independent of the pivot sequence). In our case, the width $\omega(b)$ of $b$ is $q-1$. Therefore,

$$
\lambda(b)=l(b)+i \theta(b) \doteq \frac{2 \pi i}{\omega(b)+i}=\frac{2 \pi i}{q-1+i} .
$$




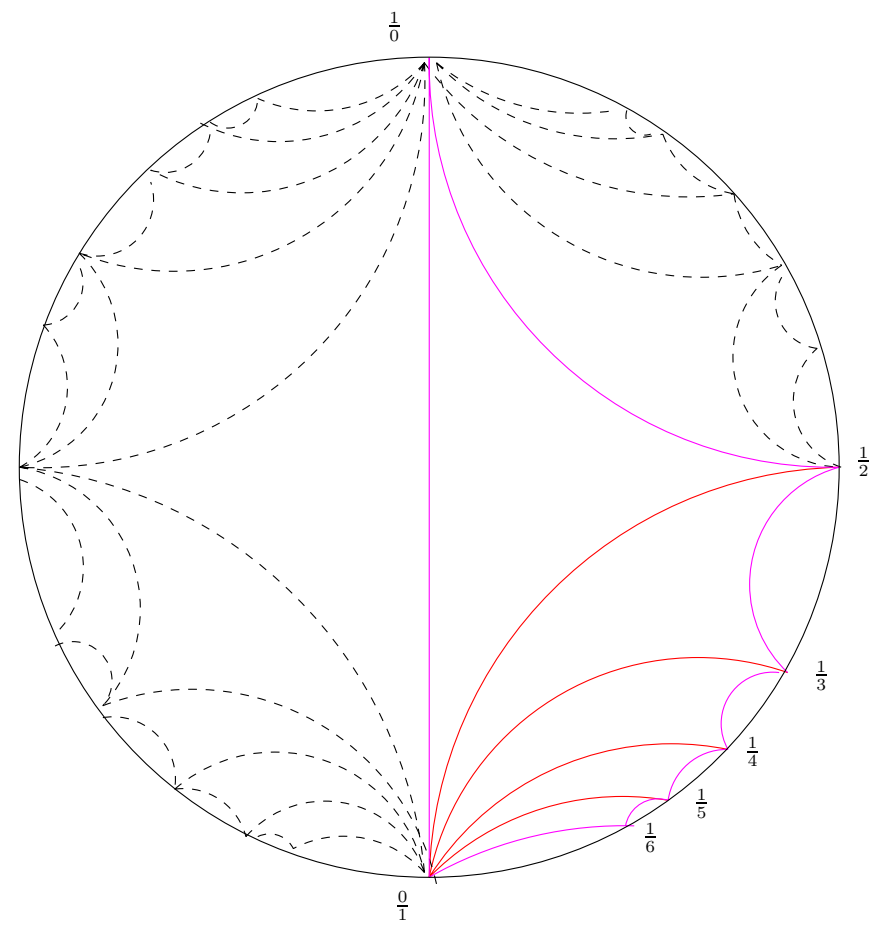

FiguRE 8. The Farey triangulation for $q=6$

We therefore obtain that universal constants $c_{1}, c_{2}, c_{3}$ exist such that

$$
\frac{c_{1}}{q^{2}} \leq l(b) \leq \frac{c_{2}}{q^{2}}
$$

and

$$
\left|q-\frac{2 \pi}{\theta(b)}\right| \leq c_{3}
$$

Equation (9) implies that

$$
\frac{2 \pi}{q+c_{3}} \leq \theta(b) \leq \frac{2 \pi}{q-c_{3}}
$$

and when $q \rightarrow \infty$,

$$
q \theta(b) \longrightarrow 2 \pi
$$

Applying Proposition 2.2, we obtain the following estimate for the radius $r(b)$ of the Margulis tube $T_{b}$ around $b$ :

$$
r(b) \sim \frac{1}{2} \log \left(\frac{1}{l(b)}\right) .
$$

To define the core chain of circles, we have to calculate the words $E_{i}$ by induction. This case is very simple since $p=1$ and therefore, following the inductive formula in Section 2, $E_{i}=b^{i}$. Therefore, the tangent circles $C_{i}$ are defined by $C_{i}=E_{i}\left(C_{0}\right)=$ $b^{i}\left(C_{0}\right)$. 


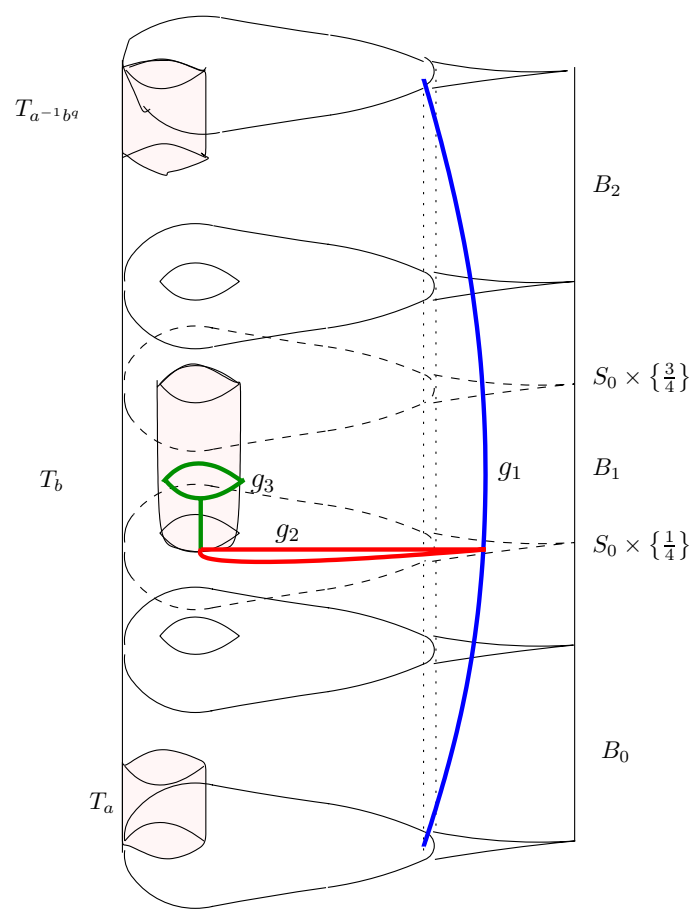

FiguRE 9. The model manifold for $p=1$

Following the parametrisation chosen in Section 2.4 for which the generators are $a=\left(\begin{array}{ll}1 & 2 \\ 0 & 1\end{array}\right)$ and $b_{\mu}=\left(\begin{array}{cc}-i \mu & -i \\ -i & 0\end{array}\right)$, the first circle $C_{0}$ is tangent to $z_{1}=-1$ to the line $\Im z=0$ in the complex plane. The second circle $C_{1}$ is tangent in $z_{2}=\mu-1$ to the line $\Im z=\Im \mu$.

Proposition 7.1. Let $V$ be the point of tangency between $C_{0}$ and $C_{1}$. Let $r_{0}$ be the radius of $C_{0}$. Then

$$
V=\frac{|\mu|}{2} e^{i \arcsin \frac{|\mu|}{4 r_{0}}}-1 .
$$

Proof. Let $E$ be the centre of $C_{0}$ and let $\eta_{1}$ be the segment between -1 and $E$. Let us denote $\eta_{2}$ the segment between $V$ and $E, L$ the distance between -1 and $V, \tau$ the angle between $\eta_{1}$ and $\eta_{2}$ and $\phi$ the angle between $L$ and the line $\Im z=0$. To clarify these notations one can see Figure 10.

Using Proposition [5.4 we have that $r_{1}=r_{0}$, where $r_{1}$ is the radius of the circle $C_{1}$.

By simple geometry, we can notice that

$$
\phi=\frac{\tau}{2}
$$

and that

$$
L=2 r_{0} \sin \left(\frac{\tau}{2}\right)=2 r_{0} \sqrt{\frac{1-\cos \tau}{2}} .
$$




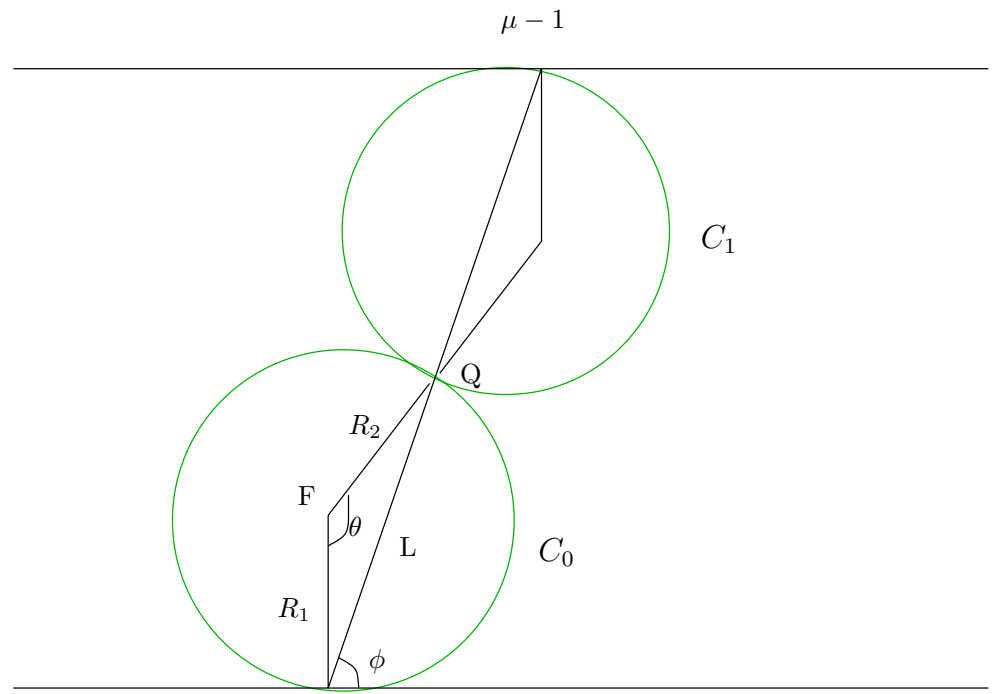

Figure 10. The tangency point between $C_{0}$ and $C_{1}$

Since the distance between the two points in the complex plane -1 and $\mu-1$ is $2 L$, we have:

$$
|\mu|=4 r_{0} \sin \frac{\tau}{2} .
$$

Therefore,

$$
\cos \tau=1-\frac{|\mu|^{2}}{8 r_{0}^{2}}
$$

and

$$
\frac{\tau}{2}=\arcsin \frac{|\mu|}{4 r_{0}}
$$

We can finally find the expression for the tangency point $V$ :

$$
V=2 r_{0} \sin \frac{\tau}{2} e^{i \phi}-1=\frac{|\mu|}{2} e^{i \arcsin \frac{|\mu|}{4 r_{0}}}-1 .
$$

Proposition 7.2. The radius of $C_{1}$ satisfies $r_{1}=O(1)$.

Proof. First we notice that the circle $C_{0}$ is contained in the band

$$
\{z \in \mathbb{C}: 0 \leq \Im z \leq \Im \mu\}
$$

and that $C_{0}$ is tangent to the line $\Im z=0$ and tangent to the circle $C_{1}$.

Moreover, the circle $C_{1}$ is tangent to the line $\Im z=\Im \mu$ and the circles $C_{0}$ and $C_{1}$ have the same radius.

Since

$$
1 \leq|\Im \mu| \leq 2,
$$

we obtain that $r\left(C_{1}\right) \sim 1$ whenever $q \gg 0$. 
7.2. Estimates of the other circles. From Theorem 5.1 and Proposition 5.4 we know that $r_{0}=r_{p}=r_{q} \sim \frac{1}{n}$. We now want to give an estimate for the other circles. In the case $p=1, n$ is fixed $(n=3)$, therefore our estimates do not depend on $n$, but they are interesting when $q$ tends to infinity. For this aim we first prove a simple remark that will be useful for our calculations.

Remark 7.3. Let $\tilde{x}=i$ and $\tilde{y}$ in the imaginary axis with $0<\tilde{y} \leq \tilde{x}$ be two points of the hyperbolic plane; let $\tilde{\gamma}$ be the geodesic between $\tilde{x}$ and $\tilde{y}$. Let $H$ be the semi-circle of radius $r$ perpendicular to the boundary of the hyperbolic plane and perpendicular to $\tilde{\gamma}$ in $\tilde{y}$.

Then $r=e^{-l(\tilde{\gamma})}$.

Proof. Let us denote $\tilde{y}=Y i$. An easy calculation shows that $l(\tilde{\gamma})=d(\tilde{x}, \tilde{y})=$ $\log \left(\frac{1}{Y}\right)$. Therefore, the Euclidean radius of $H$ satisfies the following equality:

$$
r=Y=\frac{1}{e^{l(\tilde{\gamma})}} .
$$

Theorem 7.4. The radius of $C_{k}$ such that $4<k \leq \frac{q}{2}$ satisfies $r_{k} \sim \frac{1}{e^{V_{1}}}$ where

$$
V_{1}=\frac{4 k}{\sqrt{k^{2}-1}}+\log \left(\frac{\pi^{2}\left(k^{2}-1\right)}{2 c_{4}}\right) .
$$

The constant $c_{4}$ is universal. Moreover, $r_{\frac{q}{m}}$ tends to 0 when $q \rightarrow \infty$.

Proof. For the notation we refer to Theorem 5.1 .

Let $f$ be the homotopy equivalence between $M$ and $N$, whose lift is a quasiisometry outside the Margulis tubes.

As in Theorem 5.1 we consider a point $x \in M$ in one component $\partial X^{-}$of the boundary of the convex core, such that the lift $\tilde{x} \in \mathbb{H}^{3}$ of $f(x) \in N$ satisfies $\tilde{x}=-1+j$.

Let $R$ be the disk as in Theorem 5.1 and let $H_{C_{k}}$ be the hemisphere above the circle $b^{k}\left(C_{0}\right)=C_{k}$.

We want to choose a path $\gamma_{k} \in M$ in the homotopy class of $b^{k}$ such that the lift $\widetilde{f\left(\gamma_{k}\right)} \in \mathbb{H}^{3}$ of $f\left(\gamma_{k}\right)$ starts at $\tilde{x}$ and ends in a point $\widetilde{y_{k}} \in H_{C_{k}}$.

The path $\gamma_{k}$ is the union of the sub-paths $g_{1}, g_{2}$ and $g_{3}$ that we define in what follows.

As we described in Section 2, the block $B_{1}$ contains the Margulis tube $T_{b}$ of the pivot $b$. Let $S_{0} \times\left\{\frac{1}{4}\right\}$ be the torus in $B_{1}$, with a neighbourhood of the main cusp removed. By construction, $S_{0} \times\left\{\frac{1}{4}\right\}$ contains one component of the boundary of $T_{b}$ (see Figure 9).

Let $g_{1}$ be the vertical path starting from $x$ and ending at $\partial X^{+}$, that is, a path of length $l\left(g_{1}\right)=3$, since we have 3 blocks (each block has height 1 ). Let $g_{3, k}$ be the shortest path in $T_{b}$ homotopic to $b^{k}$ with endpoints in the intersection between the boundary of the Margulis tube and $S_{0} \times\left\{\frac{1}{4}\right\}$. Let $g_{2}$ be the geodesic in $S_{0} \times\left\{\frac{1}{4}\right\}$ connecting $g_{3, k}$ with $g_{1} \cap\left(S_{0} \times\left\{\frac{1}{4}\right\}\right)$ such that $l\left(g_{2}\right) \sim 1$.

Let $y_{k} \in M$ be the endpoint of $\gamma_{k}$ in $\partial X^{+}$, let $\widetilde{f\left(\gamma_{k}\right)} \in \mathbb{H}^{3}$ be the lift of $f\left(\gamma_{k}\right)$ starting from $\tilde{x}$ and let $\widetilde{y_{k}} \in H_{C_{k}}$ be the endpoint of $\widetilde{f\left(\gamma_{k}\right)}$. Since $\gamma_{k}$ is the shortest path between $x$ and $y_{k}$ in the given homotopy class, then $\widetilde{\gamma_{k}}$ has to end in a neighbourhood of the top of $H_{C_{k}}$ (otherwise the path would be longer). 
Since we want to find the hyperbolic distance between $\tilde{x}$ and $\widetilde{y_{k}}$, we calculate the length of the geodesics $\mu_{1}, \mu_{2}$ and $\mu_{3, k}$ which have the same endpoints of $\widetilde{f\left(g_{1}\right)}$, $\widetilde{f\left(g_{2}\right)}$, and $\widetilde{f\left(g_{3, k}\right)}$, respectively.

Since $g_{1}$ is in the boundary of the main cusp, then

$$
l_{N}\left(f\left(g_{1}\right)\right) \sim l_{M}\left(g_{1}\right)=3 .
$$

Therefore, using Lemma 5.2

$$
l_{\mathbb{H}^{3}}\left(\mu_{1}\right) \sim \ln (3) \sim 1 .
$$

Since $\tilde{f}$ is a quasi-isometry outside the Margulis tubes, then

$$
l_{N}\left(f\left(g_{2}\right)\right) \sim l_{M}\left(g_{2}\right) \sim 1 .
$$

Therefore,

$$
l_{\mathbb{H}^{3}}\left(\mu_{2}\right) \sim 1 .
$$

Since $g_{3, k}$ is inside the Margulis tube $T_{b}$, then

$$
l_{\mathbb{H}^{3}}\left(\mu_{3, k}\right) \sim l_{M}\left(g_{3, k}\right),
$$

and therefore we want to find the length of $g_{3, k}$ for different $k$, with more interest when $k=1$ and $k=\frac{q}{2}$.

As we defined before, $g_{3, k}$ is the shortest path in $T_{b}$ homotopic to $b^{k}$ with endpoints in the boundary of $T_{b}: g_{3, k}$ consists of a part penetrating the tube and a loop around the tube.

Suppose that $\rho$ is the radius of the Margulis tube, that is, the distance between the boundary of $T_{b}$ and the geodesic core $b$. Let $h$ be a variable between 0 and $\rho$ and let $L(h)$ be the length of a curve homotopic to $b$ at distance $h$ from the core of $T_{b}$. Since $b$ is a pivot, then from Proposition 2.2 and from Equation (8), there exists a positive universal constant $c_{4}$ such that the radius $\rho$ satisfies the estimate:

$$
\rho \sim \frac{1}{2} \log \left(\frac{1}{l(b)}\right)=\frac{1}{2} \log \left(\frac{q^{2}}{c_{4}}\right)=\log \left(\frac{q}{\sqrt{c_{4}}}\right),
$$

therefore, $h$ varies between 0 and $\log \left(\frac{q}{\sqrt{c_{4}}}\right)$.

In Proposition 2.3 we proved the following equation (that will be useful for calculating $L(h))$ :

$$
\sinh ^{2} h=\frac{\cosh (L(h))-\cosh (l)}{\cosh (l)-\cos (\theta)} .
$$

In fact, since $g_{3, k}$ is a geodesic, then

$$
l\left(g_{3, k}\right)=\min \{F(h): F(h)=k L(h)+2(\rho-h)\} .
$$

This is the shortest path in the Model manifold homotopic to $b^{k}$ with endpoints in the intersection between the boundary of the Margulis tube and $S_{0} \times\left\{\frac{1}{4}\right\}$.

The quantity $L(h)$ describes how long the loop homotopic to $b$ is at different heights $h$ in the Margulis tube, and the term $(\rho-h)$ represents how deep we have to go inside the Margulis tube to obtain the shortest path $g_{3, k}$. Since $b$ is short, the Margulis tube could be very long and it could have a big twist (due to the rotation angle). We want to find the value of $h$ which minimises the sum of $k L(h)$ and $2(\rho-h)$.

From Equation (11),

$$
L(h)=\cosh ^{-1}\left[\cosh (l(b))\left(1+\sinh ^{2}(h)\right)-\cos (\theta(b)) \sinh ^{2}(h)\right],
$$


therefore,

$F(h)=k \cosh ^{-1}\left[\sinh ^{2}(h)(\cosh (l(b))-\cos (\theta(b)))+\cosh (l(b))\right]+2 \log \left(\frac{q}{\sqrt{c_{4}}}\right)-2 h$.

To find the minimum of $F(h)$, we calculate the derivative. To simplify the notation, let us define $D=\cosh (l(b))-\cos (\theta(b))$ and $E=\cosh (l(b))$. Then

$$
F^{\prime}(h)=\frac{2 k D \sinh (h) \cosh (h)}{\sqrt{\left(D \sinh ^{2}(h)+E\right)^{2}-1}}-2,
$$

using the formula $\frac{d}{d z}\left(\cosh ^{-1}(z)\right)=\frac{1}{\sqrt{z^{2}-1}}$.

Lemma 7.5. Let $k$ satisfy the condition $1<k \leq \frac{q}{2}$.

Then $F^{\prime}(h)=0$ if and only if either $\sinh ^{2}(h)=\frac{c_{4}^{2}}{4 \pi^{2}\left(k^{2} \pi^{2}-q^{2}\right)}$ or $\sinh ^{2}(h)=$ $\frac{q^{2}-\pi^{2} k^{2}}{\pi^{2}\left(k^{2}-1\right)}$; moreover, $\sinh ^{2}(h)=\frac{q^{2}-\pi^{2} k^{2}}{\pi^{2}\left(k^{2}-1\right)}$ is the minimum of $F(h)$.

Proof. Let us consider the case in which $1<k \leq \frac{q}{2}$. We want to find the minimum of

$$
F(h)=k L(h)+2(\rho-h)=k \cosh ^{-1}\left[D \sinh ^{2}(h)+E\right]+\log \left(\frac{1}{l(b)}\right)-2 h .
$$

We calculate the derivative of $F$ :

$$
F^{\prime}(h)=\frac{2 k D \sinh (h) \cosh (h)}{\sqrt{\left(D \sinh ^{2}(h)+E\right)^{2}-1}}-2 .
$$

If $F^{\prime}(h)=0$, then $k D \sinh (h) \cosh (h)=\sqrt{\left(\sinh ^{2}(h) D+E\right)^{2}-1}$. Squaring both sides of this equality, we obtain a second degree equation in $\sinh ^{2}(h)$ :

$$
\sinh ^{4}(h)\left(k^{2} D^{2}-D^{2}\right)+\sinh ^{2}(h)\left(k^{2} D^{2}-2 D E\right)+1-E^{2}=0
$$

which has two solutions:

$$
\sinh ^{2}(h)=\frac{2 E-k^{2} D \pm \sqrt{\left(k^{2} D-2 E\right)^{2}-4\left(1-E^{2}\right)\left(k^{2}-1\right)}}{2 D\left(k^{2}-1\right)} .
$$

From Equations (8) and (9), we can calculate the Taylor expansion of $\cosh (x)$ and $\cos (x)$, obtaining

and

$$
\cosh (l(b))=1+\frac{c_{4}^{2}}{2 q^{4}}+O\left(\frac{1}{q^{6}}\right)
$$

Hence,

$$
\cos (\theta(b))=1-\frac{4 \pi^{2}}{2 q^{2}}+O\left(\frac{1}{q^{4}}\right) .
$$

$$
D=\cosh (l(b))-\cos (\theta(b))=\frac{4 \pi^{2}}{q^{2}}+O^{+}\left(\frac{1}{q^{4}}\right)
$$

and

$$
E=\cosh (l(b))=1+\frac{c_{4}^{2}}{2 q^{4}}+O^{+}\left(\frac{1}{q^{6}}\right)
$$

Therefore,

$$
D^{2}-2 D E=\cos ^{2}(\theta(b))-\cosh ^{2}(l(b))=-\frac{8 \pi^{2}}{q^{2}}+O\left(\frac{1}{q^{4}}\right) .
$$


Using Equations (13) and (14), we obtain that

$$
\begin{gathered}
2 E-k^{2} D=2-\frac{2 \pi^{2} k^{2}}{q^{2}}+O\left(\frac{1}{q^{4}}\right) \\
\left(k^{2} D-2 E\right)^{2}-4\left(1-E^{2}\right)\left(k^{2}-1\right)=\left(2-\frac{2 \pi^{2} k^{2}}{q^{2}}\right)^{2}+O\left(\frac{1}{q^{4}}\right)
\end{gathered}
$$

and

$$
2 D\left(k^{2}-1\right)=\frac{4 \pi^{2} k^{2}}{q^{2}}-\frac{4 \pi^{2}}{q^{2}}+O\left(\frac{1}{q^{4}}\right) .
$$

Therefore, using Equations (15), (16), and (17) in (12), we obtain that $F^{\prime}(h)=0$ when either $\sinh ^{2}(h)=\frac{c_{4}^{2}}{4 \pi^{2}\left(k^{2} \pi^{2}-q^{2}\right)}$ or $\sinh ^{2}(h)=\frac{q^{2}-\pi^{2} k^{2}}{\pi^{2}\left(k^{2}-1\right)}$. Studying the sign of $F^{\prime}(h)$, we obtain that the minimum point of $F(h)$ is when $\sinh ^{2}(h)=\frac{q^{2}-\pi^{2} k^{2}}{\pi^{2}\left(k^{2}-1\right)}$.

\section{Lemma 7.6.}

(1) The radius of $C_{k}$ such that $1<k \leq \frac{q}{2}$ satisfies $r_{k} \sim \frac{1}{e^{V_{1}}}$ where

$$
V_{1}=\frac{4 k}{\sqrt{k^{2}-1}}+\log \left(\frac{\pi^{2}\left(k^{2}-1\right)}{2 c_{4}}\right) \text {. }
$$

(2) Moreover, $r_{\frac{q}{m}}$ tends to 0 when $q \rightarrow \infty$ and $m>\pi$.

Proof. Let $k$ satisfy the condition that $1<k \leq \frac{q}{2}$. We want to find the minimum of $F(h)$ in the interval $h \in\left[0, \log \left(\frac{q}{\sqrt{c_{4}}}\right)\right]$. From Lemma [7.5, the minimum of $F(h)$ is when

$$
\sinh ^{2}(h)=\frac{q^{2}-\pi^{2} k^{2}}{\pi^{2}\left(k^{2}-1\right)} .
$$

Using that $\sinh ^{-1}(z) \sim \log (2 z)$ for $|z|>1$, the minimum happens when

$$
h \sim \log \left(2 \sqrt{\frac{q^{2}-\pi^{2} k^{2}}{\pi^{2}\left(k^{2}-1\right)}}\right) .
$$

A simple calculation shows that

$$
\begin{gathered}
l\left(g_{3, k}\right) \sim k \cosh ^{-1}\left[\frac{\left(q^{2}-\pi^{2} k^{2}\right) 2 \pi^{2}}{\pi^{2} q^{2}\left(k^{2}-1\right)}+1+\frac{c_{4}^{2}}{2 q^{4}}\right]+2 \log \left(\frac{q}{\sqrt{c_{4}}}\right) \\
+-2 \log \left(2 \sqrt{\frac{q^{2}-\pi^{2} k^{2}}{\pi^{2}\left(k^{2}-1\right)}}\right) \\
\sim k \frac{4 \pi}{q} \sqrt{\frac{2 q^{2}-2 \pi^{2} k^{2}}{\pi^{2}\left(k^{2}-1\right)}}+2 \log \left(\frac{q}{\sqrt{c_{4}}}\right)-2 \log \left(2 \sqrt{\frac{q^{2}-\pi^{2} k^{2}}{\pi^{2}\left(k^{2}-1\right)}}\right) \\
\sim \frac{4 k}{\sqrt{k^{2}-1}}+\log \left(\frac{q^{2}}{c_{4}}\right)-\log \left(\frac{4 q^{2}-4 \pi^{2} k^{2}}{\pi^{2}\left(k^{2}-1\right)}\right) \\
=\frac{4 k}{\sqrt{k^{2}-1}}+\log \left(\frac{\pi^{2}\left(k^{2}-1\right)}{2 c_{4}}\right)+O\left(\frac{k}{q}\right) .
\end{gathered}
$$

Therefore,

$$
l\left(\mu_{1}\right)+l\left(\mu_{2}\right)+l\left(\mu_{3, k}\right) \sim V_{1}
$$


where

$$
V_{1}=\frac{4 k}{\sqrt{k^{2}-1}}+\log \left(\frac{\pi^{2}\left(k^{2}-1\right)}{2 c_{4}}\right) .
$$

We apply Remark 7.3, to obtain that

$$
r\left(C_{k}\right) \sim \frac{1}{e^{d\left(\tilde{x}, \widetilde{y_{k}}\right)}} \sim \frac{1}{e^{V_{1}}} .
$$

When $k=\frac{q}{m}$, with $m$ fixed and $m>\pi$, then

$$
l\left(g_{3, \frac{q}{m}}\right) \sim \log \left(\frac{q^{2}}{m^{2}}\right)+\frac{4}{m} .
$$

Therefore,

$$
l\left(\mu_{1}\right)+l\left(\mu_{2}\right)+l\left(\mu_{3, \frac{q}{m}}\right) \sim V_{2}
$$

where

$$
V_{2}=\log \left(\frac{q^{2}}{m^{2}}\right)+\frac{4}{m} .
$$

7.3. Algebraic limit versus geometric limit. In Section 5, we gave an estimate of the radii of the circles $C_{n, j}$ for the manifold $N_{n}$. On the other hand, in Section 7.1 and Section 7.2 we consider the case of $n=3$ and we define a sequence of groups $G_{q}=\left(\frac{1}{0}, \frac{1}{q}\right)$, with related manifolds $N_{q}$. In this section we want to point out that there is a big difference between the sequence of manifolds $N_{n}$ and the sequence of manifolds $N_{q}$.

We remind the reader that in the case of $G_{n}=\left(\frac{1}{0}, \frac{p_{n}}{q_{n}}\right)$, we are interested in the condition when the number $n$ of the pivots tends to infinity. Instead, in the example $G_{q}=\left(\frac{1}{0}, \frac{1}{q}\right)$, we fix $n=1$ and we consider when $q$ tends to infinity.

Now we want to see that $N_{n} \longrightarrow N_{\infty}$ algebraically and geometrically, but in the case of $G_{q}$ the algebraic limit is different from the geometric limit. The first statement follows easily from the definition of geometric limit and the construction of Minsky's model manifold.

Regarding the second statement, we want to analyze $G_{\text {alg }}^{\infty}$ and $N_{\text {geo }}^{\infty}$ where

$$
G_{q} \stackrel{a l g}{\longrightarrow} G_{a l g}^{\infty}
$$

and

$$
N_{q} \stackrel{g e o}{\longrightarrow} N_{g e o}^{\infty}
$$

so $G_{\text {alg }}^{\infty}$ is the algebraic limit and $N_{\text {geo }}^{\infty}$ is the geometric limit.

Lemma 7.7. Let $G_{\text {alg }}^{\infty}$ be the algebraic limit of $G_{q}=\left(\frac{1}{0}, \frac{1}{q}\right)$, then $G_{a l g}^{\infty}=G\left(\frac{1}{0}, \frac{0}{1}\right)$.

Proof. Let $\rho_{q}$ be the representation for $G_{q}$ :

$$
G_{q}=\rho_{q}\left(\pi_{1}(S)\right)
$$

and let $\rho$ be the representation for $G\left(\frac{1}{0}, \frac{0}{1}\right)$ :

$$
G\left(\frac{1}{0}, \frac{0}{1}\right)=\rho\left(\pi_{1}(S)\right) .
$$

We want to prove that

$$
\rho_{q}(g) \longrightarrow \rho(g) \forall g \in \pi_{1}(S) .
$$

It is enough to prove that this is true for the generators $\alpha$ and $\beta$ of $\pi_{1}(S)$. 
By definition:

$$
\rho_{q}(\alpha)=\rho(\alpha)=\left(\begin{array}{ll}
1 & 2 \\
0 & 1
\end{array}\right) .
$$

For the generator $\beta$, we have that

$$
\rho_{q}(\beta)=\left(\begin{array}{cc}
-i \mu & -i \\
-i & 0
\end{array}\right)
$$

where $\mu$ satisfies the condition

$$
\operatorname{tr}\left(a^{-1} b^{q}\right)=2 .
$$

Moreover,

$$
\rho(\beta)=\left(\begin{array}{cc}
2 & -i \\
-i & 0
\end{array}\right)
$$

Using that

$$
(\operatorname{tr}(a))^{2} *(\operatorname{tr}(b))^{2} *(\operatorname{tr}(a b))^{2}=\operatorname{tr}(a) * \operatorname{tr}(b) * \operatorname{tr}(a b),
$$

we obtain that $-i \mu \stackrel{q \rightarrow \infty}{\longrightarrow} 2$ and therefore

$$
\rho_{q}(\beta) \longrightarrow \rho(\beta) .
$$

Let $N_{a l g}^{\infty}$ denote the manifold given by

$$
N_{\text {alg }}^{\infty}=\mathbb{H}^{3} / G_{\text {alg }}^{\infty}=\mathbb{H}^{3} / G(1 / 0,0 / 1) .
$$

The model manifold $M_{a l g}^{\infty}$ associated to $N_{a l g}^{\infty}$ has only one block $B_{a, b}$ (a boundary block). The generators $a$ and $b$ (related to $\frac{1}{0}$ and $\frac{0}{1}$, respectively) are accidental parabolics.

Basically, in the algebraic limit, we completely forget about the geometric structure, and therefore we forget that the new accidental parabolic $b$ comes from the parabolic $a^{-1} b^{q}$. We will see that the geometric limit reminds us of it.

If we consider the model manifold $M_{q}$ associated to $N_{q}=\mathbb{H}^{3} / G_{q}$, we notice that $M_{q}$ has two boundary blocks $B_{1}=B_{a, b}$ and $B_{2}=B_{b, a^{-1} b^{q}}$. The elements $a$ and

$a^{-1} b^{q}$ (related to $\frac{1}{0}$ and $\frac{1}{q}$, respectively) are accidental parabolics, but the element $b$ is not an accidental parabolic. When $q$ tends to infinity, the curve associated to the element $b$ is shorter and shorter, but the second block of the model manifold does not disappear for the definition of geometric limit. Therefore the limit model manifold $M_{\text {geo }}^{\infty}$ of the geometric limit $N_{\text {geo }}^{\infty}=\mathbb{H}^{3} / G_{\text {geo }}^{\infty}$ has two blocks.

From Maskit's combination theorem, an elliptic transformation $t$ exists such that

$$
G_{g e o}^{\infty}=\left\langle G_{a l g}^{\infty}, t\right\rangle .
$$

In particular, there is a covering

$$
N_{\text {geo }}^{\infty} \longrightarrow N_{a l g}^{\infty}
$$

that is $1-1$ in $B_{1}$ and $\infty-1$ otherwise.

Therefore, $N_{\text {alg }}^{\infty}$ and $N_{\text {geo }}^{\infty}$ are different.

\section{ACKNOWLEDGMENTS}

I would like to thank Caroline Series for the stimulating and interesting conversations regarding this work. I would also like to thank David Wright for providing me with some pictures included in this article. 


\section{REFERENCES}

1. H. Akiyoshi, H. Miyachi, and M. Sakuma, A refinement of McShane's identity for quasiFuchsian punctured torus groups, Preprint.

2. J. W. Anderson and R. D. Canary, Cores of hyperbolic 3-manifolds and limits of Kleinian groups. II, J. London Math. Soc. (2) 61 (2000), no. 2, 489-505. MR1760675 (2001h:30041)

3. A. F. Beardon, The geometry of discrete groups, Graduate Texts in Mathematics, vol. 91, Springer-Verlag, New York, 1983. MR0698777 (85d:22026)

4. L. Bers, Simultaneous uniformization, Bull. Amer. Math. Soc. 66 (1960), 94-97. MR0111834 $(22: 2694)$

5. F. Bonahon, Bouts des variétés hyperboliques de dimension 3, Ann. of Math. (2) 124 (1986), no. 1, 71-158. MR0847953(88c:57013)

6. B. Bowditch, The Cannon-Thurston map for punctured-surface groups, Preprint.

7. B. H. Bowditch, Markoff triples and quasi-Fuchsian groups, Proc. London Math. Soc. (3) 77 (1998), no. 3, 697-736. MR.1643429 (99f:57014)

8. R. Evans, Strong convergence of sequences of Kleinian groups, Preprint.

9. W. J. Floyd, Group completions and limit sets of Kleinian groups, Invent. Math. 57 (1980), no. 3, 205-218. MR0568933(81e:57002)

10. T. Jørgensen, On cyclic groups of Möbius transformations, Math. Scand. 33 (1973), 250-260 (1974). MR 0348103(50:601)

11. wick, 2001), London Math. Soc. Lecture Note Ser., vol. 299, Cambridge Univ. Press, Cambridge, 2003, pp. 183-207. MR2044551 (2005a:30075)

12. T. Jørgensen and A. Marden, Algebraic and geometric convergence of Kleinian groups, Math. Scand. 66 (1990), no. 1, 47-72. MR1060898 (91f:30068)

13. L. Keen, B. Maskit, and C. Series, Geometric finiteness and uniqueness for Kleinian groups with circle packing limit sets, J. Reine Angew. Math. 436 (1993), 209-219. MR1207287 (94b:30053)

14. L. Keen and C. Series, Pleating coordinates for the Maskit embedding of the Teichmüller space of punctured tori, Topology 32 (1993), no. 4, 719-749. MR1241870 (95g:32030)

15. B. Maskit, On boundaries of Teichmüller spaces and on Kleinian groups. II, Ann. of Math. (2) 91 (1970), 607-639. MR0297993 (45:7045)

16. - On boundaries of Teichmüller spaces and on Kleinian groups. II, Ann. of Math. (2) 91 (1970), 607-639. MR0297993 (45:7045)

17. C. T. McMullen, Renormalization and 3-manifolds which fiber over the circle, Annals of Mathematics Studies, vol. 142, Princeton University Press, Princeton, NJ, 1996. MR 1401347 (97f:57022)

18. Local connectivity, Kleinian groups and geodesics on the blowup of the torus, Invent. Math. 146 (2001), no. 1, 35-91. MR1859018 (2004e:30068)

19. G. McShane, Simple geodesics and a series constant over Teichmüller space, Invent. Math. 132 (1998), no. 3, 607-632. MR1625712 (99i:32028)

20. R. Meyerhoff, A lower bound for the volume of hyperbolic 3-manifolds, Canad. J. Math. 39 (1987), no. 5, 1038-1056. MR0918586 (88k:57049)

21. Y. N. Minsky, On rigidity, limit sets, and end invariants of hyperbolic 3-manifolds, J. Amer. Math. Soc. 7 (1994), no. 3, 539-588. MR1257060 (94m:57029)

22. - The classification of punctured-torus groups, Ann. of Math. (2) 149 (1999), no. 2, 559-626. MR1689341 (2000f:30028)

23. H. Miyachi, Moduli of continuity of Cannon-Thurston maps, Spaces of Kleinian Groups (2004), London Math. Soc. Lecture Note Ser., vol. 300, Cambridge Univ. Press, Cambridge, 2004, pp. 1-26.

24. J. R. Parker, Tetrahedral decomposition of punctured torus bundles, Kleinian groups and hyperbolic 3-manifolds (Warwick, 2001), London Math. Soc. Lecture Note Ser., vol. 299, Cambridge Univ. Press, Cambridge, 2003, pp. 275-291. MR2044554 (2005g:57037)

25. I. Scorza, Fractal curves in the limit sets of simply degenerate once-punctured torus groups, Preprint.

26. W. Thurston, Hyperbolic structures on 3-manifolds, II: Surface groups and manifolds which fiber over the circle, Preprint. 
27. W. P. Thurston, Three-dimensional manifolds, Kleinian groups and hyperbolic geometry, Bull. Amer. Math. Soc. (N.S.) 6 (1982), no. 3, 357-381. MR0648524 (83h:57019)

28. D. Wright, The shape of the boundary of Maskit's embedding of the Teichmüller space of once-punctured tori, Preprint.

Dipartimento di Matematica, Università di Genova, Via Dodecaneso, 35 - 16146 GenOVA, ITALY

E-mail address: scorza@dima.unige.it 